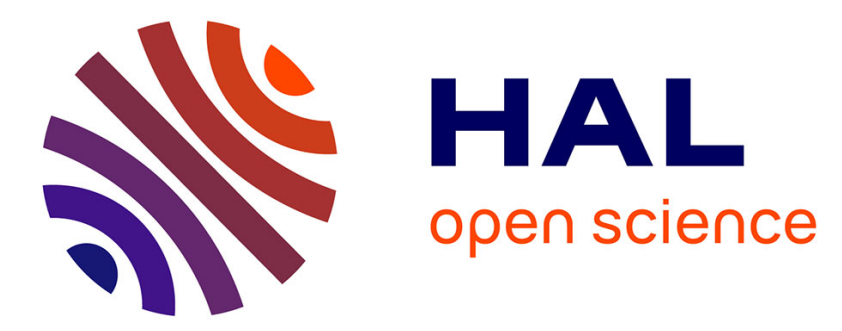

\title{
SPECIAL VALUES OF THE BELL POLYNOMIALS OF THE SECOND KIND FOR SOME SEQUENCES AND FUNCTIONS
}

Feng Qi, Da-Wei Niu, Dongkyu Lim, Yong-Hong Yao

\section{- To cite this version:}

Feng Qi, Da-Wei Niu, Dongkyu Lim, Yong-Hong Yao. SPECIAL VALUES OF THE BELL POLYNOMIALS OF THE SECOND KIND FOR SOME SEQUENCES AND FUNCTIONS. Journal of Mathematical Analysis and Applications, 2020, 491 (2), 13 p. 10.1016/j.jmaa.2020.124382 . hal$01766566 \mathrm{v} 2$

\section{HAL Id: hal-01766566 \\ https://hal.science/hal-01766566v2}

Submitted on 16 Jul 2020

HAL is a multi-disciplinary open access archive for the deposit and dissemination of scientific research documents, whether they are published or not. The documents may come from teaching and research institutions in France or abroad, or from public or private research centers.
L'archive ouverte pluridisciplinaire HAL, est destinée au dépôt et à la diffusion de documents scientifiques de niveau recherche, publiés ou non, émanant des établissements d'enseignement et de recherche français ou étrangers, des laboratoires publics ou privés. 


\title{
SPECIAL VALUES OF THE BELL POLYNOMIALS OF THE SECOND KIND FOR SOME SEQUENCES AND FUNCTIONS
}

\author{
FENG QI, DA-WEI NIU, DONGKYU LIM, AND YONG-HONG YAO \\ Dedicated to people facing and battling COVID-19
}

\begin{abstract}
In the paper, after concisely surveying some closed formulas and applications of special values of the Bell polynomials of the second kind for some special sequences and elementary functions, the authors newly establish some closed formulas for some special values of the Bell polynomials of the second kind.
\end{abstract}

\section{Contents}

1. Survey of closed formulas for the Bell polynomials 2

1.1. Exponential function 2

$\begin{array}{lll}1.2 . & \text { Logarithmic function } & 3\end{array}$

1.3. Power function 3

1.4. A sequence related to square 4

1.5. Square roots 4

1.6. Sine and cosine 6

\begin{tabular}{lll}
\hline 1.7. & Two sequences related to factorials & 7
\end{tabular}

$\begin{array}{lll}1.8 . & \text { Two simple sequences } & 7\end{array}$

$\begin{array}{lll}\text { 1.9. A sequence containing factorials } & 7\end{array}$

$\begin{array}{lll}1.10 . & \text { A sequence from natural numbers } & 8\end{array}$

1.11. Several sequences related to factorials 8

1.12. Several sequences from natural numbers 8

1.13. Howard's numbers 9

2. Closed formulas for some numbers discussed by Howard 10

3. A closed formula for a special Bell polynomial 20

$\begin{array}{lll}4 . & \text { Remarks and comparisons } & 21\end{array}$

Funding 24

Acknowledgements 24

References 24

In this paper, we have three aims which can be concretely stated as follows.

2010 Mathematics Subject Classification. Primary 11B83; Secondary 11C08, 11S05, 11T06, 12D05, 12E10, 12Y05, 13M10, 13P05, 26C05.

Key words and phrases. survey; closed formula; special value; Bell polynomial of the second kind; sequence; elementary function; application.

Please cite this article as "Feng Qi, Da-Wei Niu, Dongkyu Lim, and Yong-Hong Yao, Special values of the Bell polynomials of the second kind for some sequences and functions, Journal of Mathematical Analysis and Applications 491 (2020), no. 2, Article 124382, 31 pages; available online at https://doi.org/10.1016/j.jmaa.2020.124382"

Please download the formally published version of this article freely from https://authors. elsevier.com/a/1bPPT, WNxgTqe before 3 September 2020.

This paper was typeset using $\mathcal{A} \mathcal{M} \mathcal{S}$-LATEX. 
(1) In the first section, we will concisely survey some closed formulas and applications of special values of the Bell polynomials of the second kind for some special sequences and elementary functions. Most of these results are formally published and applied in recent years.

(2) In the second section, we will discover or recover some relations and closed formulas for those numbers discussed in 20] by Howard.

(3) In the third section, we will establish a closed formula for a special Bell polynomial of the second kind which was mentioned in 20] by Howard.

Finally, in the fourth section, we will simply compare our main results with some known results.

\section{Survey of Closed formulas for the Bell polynomials}

In [6, Definition 11.2] and [7, p. 134, Theorem A], the Bell polynomials of the second kind, denoted by $\mathrm{B}_{n, k}\left(x_{1}, x_{2}, \ldots, x_{n-k+1}\right)$ for $n \geq k \geq 0$, are defined by

$$
\mathrm{B}_{n, k}\left(x_{1}, x_{2}, \ldots, x_{n-k+1}\right)=\sum_{\substack{1 \leq i \leq n-k+1 \\ \ell_{i} \in\{0\} \cup \mathbb{N} \\ \sum_{i=k+1}^{n-k+1} i \ell_{i}=n \\ \sum_{i=1}^{n-k+1} \ell_{i}=k}} \frac{n !}{\prod_{i=1}^{n-k+1} \ell_{i} !} \prod_{i=1}^{n-k+1}\left(\frac{x_{i}}{i !}\right)^{\ell_{i}} .
$$

The Faà di Bruno formula, see [6, Theorem 11.4] and [7, p. 139, Theorem C], can be described in terms of $\mathrm{B}_{n, k}\left(x_{1}, x_{2}, \ldots, x_{n-k+1}\right)$ by

$$
\frac{\mathrm{d}^{n}}{\mathrm{~d} x^{n}} f \circ h(x)=\sum_{k=0}^{n} f^{(k)}(h(x)) \mathrm{B}_{n, k}\left(h^{\prime}(x), h^{\prime \prime}(x), \ldots, h^{(n-k+1)}(x)\right) .
$$

The identity

$$
\mathrm{B}_{n, k}\left(a b x_{1}, a b^{2} x_{2}, \ldots, a b^{n-k+1} x_{n-k+1}\right)=a^{k} b^{n} \mathrm{~B}_{n, k}\left(x_{1}, x_{2}, \ldots, x_{n-k+1}\right)
$$

for $n \geq k \geq 0$ and $a, b \in \mathbb{C}$ can be found in [6, p. 412] and [7, p. 135].

There have been a number of literature, such as [1, 6, 7, 20, 22, 23, 26, 27, 102, 103, 104, 105, dedicated to the general theory of the Bell polynomials of the second kind $\mathrm{B}_{n, k}\left(x_{1}, x_{2}, \ldots, x_{n-k+1}\right)$.

In mathematics, a closed form is a mathematical expression that can be evaluated in a finite number of operations. It may contain constants, variables, four arithmetic operations, and elementary functions, but usually no limit.

In this paper, we pay our main attention on closed and explicit formulas, rather than recursive identities like 1.2 , of the Bell polynomials of the second kind $\mathrm{B}_{n, k}\left(x_{1}, x_{2}, \ldots, x_{n-k+1}\right)$ for $x_{1}, x_{2}, \ldots, x_{n-k+1}$ to be replaced by some special sequences and elementary functions.

We now concisely survey some formulas and applications of the polynomials $\mathrm{B}_{n, k}\left(x_{1}, x_{2}, \ldots, x_{n-k+1}\right)$ for $x_{1}, x_{2}, \ldots, x_{n-k+1}$ to be replaced by some special sequences and elementary functions.

1.1. Exponential function. To find general formulas of the $n$th derivatives for functions of the type $f\left(e^{x}\right)$, such as $\frac{1}{e^{ \pm x} \pm 1}$, by the Faà di Bruno formula [1.1), we need to compute

$$
\begin{gathered}
\mathrm{B}_{n, k}\left(\left(e^{ \pm x}\right)^{\prime},\left(e^{ \pm x}\right)^{\prime \prime}, \ldots,\left(e^{ \pm x}\right)^{(n-k+1)}\right) \\
=\mathrm{B}_{n, k}\left( \pm e^{ \pm x},( \pm 1)^{2} e^{ \pm x}, \ldots,( \pm 1)^{n-k+1} e^{ \pm x}\right) \\
=( \pm 1)^{n} e^{ \pm k x} \mathrm{~B}_{n, k}(1,1, \ldots, 1),
\end{gathered}
$$

where we used in the last step the identity (1.2). In [7, p. 135], it is given that

$$
\mathrm{B}_{n, k}(1,1, \ldots, 1)=S(n, k),
$$


where $S(n, k)$ denotes the Stirling numbers of the second kind. Consequently, by the Faà di Bruno formula $(1.1)$ and the identities $\sqrt{1.2}$ and $\sqrt{1.3}$, for example, we can easily obtain

$$
\left(\frac{1}{e^{ \pm x} \pm 1}\right)^{(n)}=( \pm 1)^{n} \sum_{k=0}^{n}(-1)^{k} k ! S(n, k) \frac{e^{ \pm k x}}{\left(e^{ \pm x} \pm 1\right)^{k+1}} .
$$

For more information on applications of the identity 1.3 , please read the papers $[11,12,13,15,18,30,32,35,45,50,51,60,63,73,74,79,84,100,101,106$, [107, 109] and closely related references therein.

1.2. Logarithmic function. To acquire general formulas of the $n$th derivatives for functions of the type $f(\ln x)$, such as $\frac{1}{\ln (1+x)}$, by the Faà di Bruno formula (1.1) and the identity 1.2 , we need to compute

$$
\begin{gathered}
\mathrm{B}_{n, k}\left([\ln (1+x)]^{\prime},[\ln (1+x)]^{\prime \prime}, \ldots,[\ln (1+x)]^{(n-k+1)}\right) \\
=\mathrm{B}_{n, k}\left(\frac{1}{1+x}, \frac{-1}{(1+x)^{2}}, \ldots, \frac{(-1)^{n-k}(n-k) !}{(1+x)^{n-k+1}}\right) \\
=\frac{(-1)^{n-k}}{(1+x)^{n}} \mathrm{~B}_{n, k}(0 !, 1 !, \ldots,(n-k) !) .
\end{gathered}
$$

In [7, p. 135], it is listed that

$$
\mathrm{B}_{n, k}(0 !, 1 !, 2 !, \ldots,(n-k) !)=(-1)^{n-k} s(n, k),
$$

where $s(n, k)$ denotes the Stirling numbers of the first kind. Consequently, by virtue of the Faà di Bruno formula $\sqrt{1.1}$ and the identities $\sqrt{1.2}$ and $(1.4)$, as an example, we have

$$
\left[\frac{1}{\ln (1+x)}\right]^{(n)}=\frac{1}{(1+x)^{n}} \sum_{k=0}^{n} \frac{(-1)^{k} k ! s(n, k)}{\ln ^{k+1}(1+x)} .
$$

For more information on applications of the identity $(1.4$, please refer to [29, 38, 39, 41, 42, 44, 46, 73, 80, 91, 95, 96, and closely related references therein.

1.3. Power function. To establish general formulas of the $n$th derivatives for functions of the type $f\left(x^{\alpha}\right)$ for $\alpha \in \mathbb{R}$, such as $e^{x^{2}}$ and $\sin \sqrt{a+b x}$, by the Faà di Bruno formula (1.1) and the identity 1.2 , we need to calculate

$$
\mathrm{B}_{n, k}\left(\left(x^{\alpha}\right)^{\prime},\left(x^{\alpha}\right)^{\prime \prime}, \ldots,\left(x^{\alpha}\right)^{(n-k+1)}\right)=x^{k \alpha-n} \mathrm{~B}_{n, k}\left(\langle\alpha\rangle_{1},\langle\alpha\rangle_{2}, \ldots,\langle\alpha\rangle_{n-k+1}\right),
$$

where

$$
\langle\alpha\rangle_{n}=\prod_{k=0}^{n-1}(\alpha-k)= \begin{cases}\alpha(\alpha-1) \cdots(\alpha-n+1), & n \geq 1 \\ 1, & n=0\end{cases}
$$

is called [87] the falling factorial. In 83], it was discovered that

$$
\mathrm{B}_{n, k}\left(\langle\alpha\rangle_{1},\langle\alpha\rangle_{2}, \ldots,\langle\alpha\rangle_{n-k+1}\right)=\frac{(-1)^{k}}{k !} \sum_{\ell=0}^{k}(-1)^{\ell}\left(\begin{array}{l}
k \\
\ell
\end{array}\right)\langle\alpha \ell\rangle_{n} .
$$

The formula 1.5 has an equivalent form

$$
\begin{aligned}
\mathrm{B}_{n, k}\left(1,1-\lambda,(1-\lambda)(1-2 \lambda), \ldots, \prod_{\ell=0}^{n-k}(1-\ell \lambda)\right) & \\
= & \frac{(-1)^{k}}{k !} \sum_{\ell=0}^{k}(-1)^{\ell}\left(\begin{array}{c}
k \\
\ell
\end{array}\right) \prod_{q=0}^{n-1}(\ell-q \lambda)
\end{aligned}
$$


which was earlier obtained in [72]. For more information on applications of the formula 51.6, , please see [49, 55, 56, 67, 72, 76, 77, 81, 83] and closely related references.

Taking $\lambda=0$ in (1.6) recovers the identity 1.3 . Letting $\lambda=-1$ in 1.6 and simplifying give

$$
\begin{aligned}
\mathrm{B}_{n, k}(1 !, 2 !, 3 !, \ldots,(n-k+1) !) & =(-1)^{k} \frac{n !}{k !} \sum_{\ell=1}^{k}(-1)^{\ell}\left(\begin{array}{c}
k \\
\ell
\end{array}\right)\left(\begin{array}{c}
n+\ell-1 \\
\ell-1
\end{array}\right) \\
& =\left(\begin{array}{c}
n-1 \\
k-1
\end{array}\right) \frac{n !}{k !}
\end{aligned}
$$

which is the Lah numbers collected in [6, p. 450] and [7, p. 135]. For more information on applications of the formula (1.7), please refer to the papers [17, 33, 37, 39, 47, 48, 59, 62, 66, 69, 71, 72, 73, 77, 83, 87, 88, 93. and closely related references therein.

By the way, the $n$th derivatives for functions of the type $f\left(x^{n}\right)$ were recursively, not explicitly, discussed in [27, Section 4].

1.4. A sequence related to square. In [65, Theorem 5.1] and [97, Section 3], it was found little by little that

$$
\mathrm{B}_{n, k}(x, 1,0, \ldots, 0)=\frac{1}{2^{n-k}} \frac{n !}{k !}\left(\begin{array}{c}
k \\
n-k
\end{array}\right) x^{2 k-n},
$$

where $\left(\begin{array}{l}0 \\ 0\end{array}\right)=1$ and $\left(\begin{array}{l}p \\ q\end{array}\right)=0$ for $q>p \geq 0$. Consequently, as an example, we have

$$
\left(e^{x^{2}}\right)^{(n)}=e^{x^{2}} \frac{n !}{(2 x)^{n}} \sum_{k=0}^{n}\left(\begin{array}{c}
k \\
n-k
\end{array}\right) \frac{(2 x)^{2 k}}{k !} .
$$

Taking $\alpha=2$ in 1.5 or taking $\lambda=\frac{1}{2}$ in 1.6, making use of 1.2 , letting $x=1$ in 1.8 , and comparing yield the sum

$$
\sum_{\ell=0}^{k}(-1)^{k-\ell}\left(\begin{array}{l}
k \\
\ell
\end{array}\right)\langle 2 \ell\rangle_{n}=\frac{n !}{2^{n-2 k}}\left(\begin{array}{c}
k \\
n-k
\end{array}\right) .
$$

One of anonymous referees pointed out that the formula 1.8 is essentially the same as those formulas in [26. Section 4]. The difference is that, in the article [26], the expressions are given, in compact form, in terms of the Hermite-Kampé de Fériet polynomials. By using definitions of these classical polynomials [3], the explicit forms given in 1.8 can be recovered.

For detailed information on applications of the formula $(1.8)$, please refer to the papers [31, 40, 54, 56, 62, 65, 67, 70, 72, 82, 83, 86, 88, 97, 98, 101, 111] and closely related references therein.

1.5. Square roots. In [88, Theorem 1.4], it was presented that

$$
\begin{aligned}
& \mathrm{B}_{n, k}\left(g^{\prime}(x), g^{\prime \prime}(x), \ldots, g^{(n-k+1)}(x)\right) \\
&=(-1)^{n+k}[2(n-k)-1] ! !\left(\frac{b}{2}\right)^{n}\left(\begin{array}{c}
2 n-k-1 \\
2(n-k)
\end{array}\right) \frac{1}{(a+b x)^{n-k / 2}},
\end{aligned}
$$

where $g(x)=\sqrt{a+b x}$ for $a, b \in \mathbb{R}$ and $b \neq 0$.

Setting $a=0$ and $b=1$ and letting $x \rightarrow 1$ in 1.9 result in

$$
\mathrm{B}_{n, k}((-1) ! !, 1 ! !, 3 ! !, \ldots,[2(n-k)-1] ! !)=[2(n-k)-1] ! !\left(\begin{array}{c}
2 n-k-1 \\
2(n-k)
\end{array}\right),
$$


where the double factorial of negative odd integers $-(2 \ell+1)$ is defined by

$$
(-2 \ell-1) ! !=\frac{(-1)^{\ell}}{(2 \ell-1) ! !}=(-1)^{\ell} \frac{2^{\ell} \ell !}{(2 \ell) !}, \quad \ell \geq 0 .
$$

Taking $\alpha=\frac{1}{2}$ in 1.5 or taking $\lambda=2$ in (1.6), simplifying, and comparing with 1.10 arrive at the sum

$$
\sum_{\ell=0}^{k}(-1)^{\ell}\left(\begin{array}{l}
k \\
\ell
\end{array}\right)\left\langle\frac{\ell}{2}\right\rangle_{n}=(-1)^{n} \frac{k ![2(n-k)-1] ! !}{2^{n}}\left(\begin{array}{c}
2 n-k-1 \\
2(n-k)
\end{array}\right) .
$$

By virtue of the formula (1.1), the identity 1.2 , and the formula 1.10 in sequence, we derived in 83 five closed formulas for the (modified) spherical Bessel functions $\mathrm{j}_{n}(z), \mathrm{y}_{n}(z), \mathrm{i}_{n}^{(1)}(z), \mathrm{i}_{n}^{(2)}(z)$, and $\mathrm{k}_{n}(z)$, whose definitions can be found in [28, p. 262-266], from considering the $n$th derivatives of the functions

$$
\begin{gathered}
\cos \sqrt{z^{2}-2 z t}, \quad \sin \sqrt{z^{2}-2 z t}, \quad \cosh \sqrt{z^{2}+2 i z t}, \\
\sinh \sqrt{z^{2}+2 i z t}, \quad \exp \left(-\sqrt{z^{2}+2 i z t}\right)
\end{gathered}
$$

with respect to $t$.

In [8], it was obtained that

$$
\frac{\mathrm{d}^{n}\left(e^{s \sqrt{x}}\right)}{\mathrm{d} x^{n}}=\left(\frac{s}{2 \sqrt{x}}\right)^{n} e^{s \sqrt{x}} \sum_{k=0}^{n-1}\left(\frac{1}{s \sqrt{x}}\right)^{k} \frac{(-1)^{k}(n+k-1) !}{2^{k} k !(n-k-1) !}
$$

and

where $n \geq 1, x>0$, and

$$
K_{n-1 / 2}(-\sqrt{x})=\sqrt{\pi}(-1)^{-1 / 2}(2 \sqrt{x})^{n-1 / 2}\left(e^{\sqrt{x}}\right)^{(n)}
$$

$$
K_{n+1 / 2}(z)=\sqrt{\frac{\pi}{2 z}} e^{-z} \sum_{k=0}^{n} \frac{(n+k) !}{k !(n-k) !(2 z)^{k}}, \quad z \in \mathbb{C}
$$

is known as the modified spherical Bessel functions of the third kind 2, p. 444, 10.2.15]. In [25], it was further established that

$$
\begin{aligned}
I_{n-1 / 2}^{(\ell)}(-\sqrt{x}) & =\frac{(-1)^{(-1)^{n-1} / 2}}{\sqrt{\pi}}(2 \sqrt{x})^{n-1 / 2}\left[e^{(-1)^{\ell} \sqrt{x}}\right]^{(n)}, \\
H_{n-1 / 2}^{(\ell)}(-i \sqrt{x}) & =\frac{2(-1)^{(-1)^{n-1} / 2}}{\sqrt{\pi}}(2 i \sqrt{x})^{n-1 / 2}\left[e^{(-1)^{\ell-1} \sqrt{x}}\right]^{(n)},
\end{aligned}
$$

and

$$
H_{n-1 / 2}^{(2)}(i \sqrt{x})=\frac{2}{\sqrt{\pi}}(2 i \sqrt{x})^{n-1 / 2}\left(e^{\sqrt{x}}\right)^{(n)}
$$

for $i=\sqrt{-1}$ and $\ell=1,2$, where

$$
I_{n+1 / 2}^{(\ell)}(z)=\frac{(-1)^{(\ell-1)(n+1)}}{\sqrt{2 \pi z}} e^{(-1)^{\ell-1} z} \sum_{k=0}^{n} \frac{(-1)^{\ell k}(n+k) !}{k !(n-k) !(2 z)^{k}}
$$

for $z \in \mathbb{C}$ and $\ell=1,2$, which can be derived from a combination of [2, p. 443, 10.2.9 and 10.2.11], are known as the modified spherical Bessel functions of the first kind, and

$$
H_{n-1 / 2}^{(\ell)}(z)=\sqrt{\frac{2}{\pi z}} i^{(-1)^{\ell} n} e^{(-1)^{\ell-1} i z} \sum_{k=0}^{n-1}(-1)^{\ell k} \frac{(n+k-1) !}{k !(n-k-1) !(2 i z)^{k}}
$$

for $z \in \mathbb{C}$ and $\ell=1,2$, which is a combination of the formulas (1.7) and (1.8) in 25], are known as the Hankel functions. By virtue of the Faà di Bruno formula (1.1) and the formula $\sqrt{1.9}$ for $a=b=1$, we can simply recover the formula (1.11). 
The modified Bessel functions of the first kind, which are related to the modified spherical Bessel functions mentioned above, were applied and studied in [19, 43, 52, 89, 90, 94. For more information on applications of the formulas $(1.9)$ and $\sqrt{1.10}$, please refer to the papers [49, 68, 73, 83, 93, 88, and closely related references therein.

1.6. Sine and cosine. In [34, Theorem 1.2], it was derived that

$$
\begin{aligned}
& \mathrm{B}_{n, k}\left(-\sin x,-\cos x, \sin x, \cos x, \ldots, \cos \left[x+\frac{(n-k+1) \pi}{2}\right]\right) \\
& =\frac{(-1)^{k} \cos ^{k} x}{k !} \sum_{\ell=0}^{k}\left(\begin{array}{l}
k \\
\ell
\end{array}\right) \frac{(-1)^{\ell}}{(2 \cos x)^{\ell}} \sum_{q=0}^{\ell}\left(\begin{array}{l}
\ell \\
q
\end{array}\right)(2 q-\ell)^{n} \cos \left[(2 q-\ell) x+\frac{n \pi}{2}\right]
\end{aligned}
$$

and

$$
\begin{aligned}
& \mathrm{B}_{n, k}\left(\cos x,-\sin x,-\cos x, \sin x, \ldots, \sin \left[x+\frac{(n-k+1) \pi}{2}\right]\right) \\
= & \frac{(-1)^{k} \sin ^{k} x}{k !} \sum_{\ell=0}^{k}\left(\begin{array}{l}
k \\
\ell
\end{array}\right) \frac{1}{(2 \sin x)^{\ell}} \sum_{q=0}^{\ell}(-1)^{q}\left(\begin{array}{l}
\ell \\
q
\end{array}\right)(2 q-\ell)^{n} \cos \left[(2 q-\ell) x+\frac{(n-\ell) \pi}{2}\right] .
\end{aligned}
$$

Since $\sin \left(x \pm \frac{\pi}{2}\right)= \pm \cos x$ and $\cos \left(x \pm \frac{\pi}{2}\right)=\mp \sin x$, the formulas 1.12 and 1.13 are equivalent to each other. These two formulas can be applied to establish general formulas of the $n$th derivatives for functions of the types $f(\sin x)$ and $f(\cos x)$, such as $\sin ^{\alpha} x, \cos ^{\alpha} x, \sec ^{\alpha} x, \csc ^{\alpha} x, e^{ \pm \sin x}, e^{ \pm \cos x}, \ln \cos x, \ln \sin x, \ln \sec x, \ln \csc x$, $\sin \sin x, \cos \sin x, \sin \cos x, \cos \cos x, \tan x$, and $\cot x$. For more information on related applications and approaches, please refer to the papers [34, 57, 61, 101, 106, 108 and closely related references therein.

Recall from [4, p. 243, Eq. (10) and p. 250, Theorem 16] and [5, p. 152, Eq. (3.9)] that weighted Stirling numbers, whose definitions can be found in [4, 5, 9, can be generated by

$$
\frac{\left(e^{t}-1\right)^{k}}{k !} e^{r t}=\sum_{n=0}^{\infty} S_{r}(n, k) \frac{t^{n}}{n !}=\sum_{n=k}^{\infty} S_{r}(n, k) \frac{t^{n}}{n !} .
$$

Recall from [5, p. 152, Eq. (3.8)] that

$$
S_{r}(n, k)=\frac{1}{k !} \sum_{j=0}^{k}(-1)^{k-j}\left(\begin{array}{l}
k \\
j
\end{array}\right)(r+j)^{n}, \quad n \geq k \geq 0 .
$$

It is clear that $S_{0}(n, k)=S(n, k)$ and $S_{r}(n, m)=0$ for $m>n$. See also [10, 65. and closely related reference therein. Taking $x \rightarrow 0$ in 1.12 and $(1.13)$ leads to

$$
\begin{aligned}
\mathrm{B}_{n, k}(0,-1,0,1, \ldots, & \left.\cos \frac{(n-k+1) \pi}{2}\right) \\
= & \frac{(-1)^{k}}{k !}\left(\cos \frac{n \pi}{2}\right) \sum_{\ell=0}^{k} \frac{(-1)^{\ell}}{2^{\ell}}\left(\begin{array}{l}
k \\
\ell
\end{array}\right) \sum_{q=0}^{\ell}\left(\begin{array}{l}
\ell \\
q
\end{array}\right)(2 q-\ell)^{n}
\end{aligned}
$$

and

$$
\begin{aligned}
\mathrm{B}_{n, k}\left(1,0,-1,0, \ldots, \sin \frac{(n-k+1) \pi}{2}\right) & \\
= & \frac{(-1)^{k}}{k ! 2^{k}}\left[\cos \frac{(n-k) \pi}{2}\right] \sum_{q=0}^{k}(-1)^{q}\left(\begin{array}{l}
k \\
q
\end{array}\right)(2 q-k)^{n}
\end{aligned}
$$




$$
=\left[\cos \frac{(n-k) \pi}{2}\right] 2^{n-k} S_{-k / 2}(n, k) .
$$

Similar to the formulas (1.14 and 1.15), another two formulas

$$
\begin{aligned}
\mathrm{B}_{n, k}\left(1,0,1, \ldots, \frac{1-(-1)^{n-k+1}}{2}\right) & =\frac{1}{2^{k} k !} \sum_{\ell=0}^{k}(-1)^{\ell}\left(\begin{array}{c}
k \\
\ell
\end{array}\right)(k-2 \ell)^{n} \\
= & (-1)^{n-k} 2^{n-k} S_{-k / 2}(n, k)=2^{n-k} S_{-k / 2}(n, k)
\end{aligned}
$$

and

$$
\begin{aligned}
\mathrm{B}_{n, k}\left(0,1,0, \ldots, \frac{1+(-1)^{n-k+1}}{2}\right) & =\frac{1}{2^{k} k !} \sum_{\ell=0}^{2 k}(-1)^{\ell}\left(\begin{array}{c}
2 k \\
\ell
\end{array}\right)(k-\ell)^{n} \\
& =(-1)^{n} \frac{(2 k) !}{(2 k) ! !} S_{-k}(n, 2 k)
\end{aligned}
$$

where $0^{0}$ is regarded as 1 , were established, discussed, and applied to derive a closed formula for the Euler numbers in [65, 92, 101].

1.7. Two sequences related to factorials. In [37, Theorem 1.1], the formulas

$$
\mathrm{B}_{n, k}\left(\frac{1 !}{2}, \frac{2 !}{3}, \ldots, \frac{(n-k+1) !}{n-k+2}\right)=(-1)^{n-k} \frac{1}{k !} \sum_{m=0}^{k}(-1)^{m}\left(\begin{array}{c}
k \\
m
\end{array}\right) \frac{s(n+m, m)}{\left(\begin{array}{c}
n+m \\
m
\end{array}\right)}
$$

and

$$
\mathrm{B}_{n, k}(0,1 !, 2 ! \ldots,(n-k) !)=(-1)^{n}\left(\begin{array}{l}
n \\
k
\end{array}\right) \sum_{\ell=0}^{k}(-1)^{\ell} \frac{\left(\begin{array}{c}
k \\
\ell
\end{array}\right)}{\left(\begin{array}{c}
n-k+\ell \\
\ell
\end{array}\right)} s(n-k+\ell, \ell)
$$

were proved for $n \geq k \geq 1$. These two formulas were used in [37, 59] to discover diagonal recurrence relations for the Stirling numbers of the first kind.

1.8. Two simple sequences. In [14, 16, 36, 53, 58, 110, the formulas

$$
\mathrm{B}_{n, k}(0,1, \ldots, 1)=\sum_{\ell=0}^{k}(-1)^{\ell}\left(\begin{array}{l}
n \\
\ell
\end{array}\right) S(n-\ell, k-\ell)
$$

and

$$
\mathrm{B}_{n, k}\left(\frac{1}{2}, \frac{1}{3}, \ldots, \frac{1}{n-k+2}\right)=\frac{n !}{(n+k) !} \sum_{\ell=0}^{k}(-1)^{k-\ell}\left(\begin{array}{c}
n+k \\
k-\ell
\end{array}\right) S(n+\ell, \ell)
$$

were established and applied to investigate functions of the type $f\left(\frac{e^{t}-1}{t}\right)$ such as generating functions for the Bernoulli numbers and polynomials and for the Stirling numbers and polynomials.

1.9. A sequence containing factorials. In [86, Lemma 6], among other things, the formula

$$
\mathrm{B}_{n, k}(2 !, 3 !, \ldots,(n-k+2) !)=\frac{n !}{k !} \sum_{\ell=0}^{k}(-1)^{k-\ell}\left(\begin{array}{c}
k \\
\ell
\end{array}\right)\left(\begin{array}{c}
n+2 \ell-1 \\
n
\end{array}\right),
$$

where $\left(\begin{array}{l}q \\ 0\end{array}\right)=1$ for all $q \in \mathbb{C}$, was deduced for establishing a closed formula for the large Schröder numbers. See also 62 and closely related references therein. 
1.10. A sequence from natural numbers. In [64, Lemma 2], the formula

$$
\begin{aligned}
\mathrm{B}_{n, k}(x, 1+ & x, 2+x, \ldots, n-k+x) \\
& =k^{n-k}\left(\begin{array}{l}
n \\
k
\end{array}\right) \sum_{\ell=0}^{k}\left(\begin{array}{l}
k \\
\ell
\end{array}\right)\left[\sum_{q=0}^{n-k} \frac{(-1)^{q}}{k^{q}}\left(\begin{array}{c}
n-k \\
q
\end{array}\right) \frac{S(\ell+q, \ell)}{\left(\begin{array}{c}
\ell+q \\
\ell
\end{array}\right)}\right](x-1)^{\ell}
\end{aligned}
$$

was proved and applied to acquire closed formulas for derangement numbers and their generating function. From (1.17), the formula

$$
\mathrm{B}_{n, k}(1,2,3, \ldots, n-k+1)=\left(\begin{array}{l}
n \\
k
\end{array}\right) k^{n-k}
$$

listed in [6, p. 451] and [7, p. 135] can be recovered straightforwardly.

1.11. Several sequences related to factorials. In [6, p. 451], the formulas

$$
\begin{aligned}
\mathrm{B}_{2 r, k}(0,2 !, \ldots, 0,(2 r) !) & =\frac{(2 r) !}{k !}\left(\begin{array}{c}
r-1 \\
k-1
\end{array}\right), \\
\mathrm{B}_{2 r-1, k}(0,2 !, \ldots,(2 r-2) !, 0) & =0, \\
\mathrm{~B}_{2 r, 2 s}(1 !, 0, \ldots,(2 r-1) !, 0) & =\frac{(2 r) !}{(2 s) !}\left(\begin{array}{c}
r+s-1 \\
2 s-1
\end{array}\right), \\
\mathrm{B}_{2 r, 2 s-1}(1 !, 0, \ldots,(2 r-1) !, 0) & =0, \\
\mathrm{~B}_{2 r-1,2 s-1}(1 !, 0, \ldots,(2 r-1) !, 0) & =\frac{(2 r-1) !}{(2 s-1) !}\left(\begin{array}{c}
r+s-2 \\
2 s-2
\end{array}\right), \\
\mathrm{B}_{2 r-1,2 s}(1 !, 0, \ldots, 0,(2 r-1) !) & =0
\end{aligned}
$$

were stated as exercises. These six formulas can be uniformly written as

$$
\mathrm{B}_{n, k}\left(0,2 !, 0,4 !, \ldots,(n-k+1) ! \frac{1+(-1)^{n-k+1}}{2}\right)=\frac{1+(-1)^{n}}{2} \frac{n !}{k !}\left(\begin{array}{c}
\frac{n}{2}-1 \\
k-1
\end{array}\right)
$$

and

$$
\mathrm{B}_{n, k}\left(1 !, 0,3 !, 0, \ldots,(n-k+1) ! \frac{1-(-1)^{n-k+1}}{2}\right)=\frac{1+(-1)^{n+k}}{2} \frac{n !}{k !}\left(\begin{array}{c}
\frac{n+k}{2}-1 \\
k-1
\end{array}\right)
$$

For details on discussion of these quantities, please refer to [70, Lemma 2.5], 775] and closely related references therein.

1.12. Several sequences from natural numbers. A class of the Bell polynomials of the second kind

$$
\mathrm{B}_{n, k}\left(a_{1}, a_{2}, 3-\alpha, 4-\alpha, \ldots,(n-k+1)-\alpha\right)
$$

were investigated in [20, Section 5]. In [20, the following closed formulas were presented:

$$
\begin{aligned}
\mathrm{B}_{n, k}(0,2,3, \ldots, n-k+1) & =\frac{n !}{(n-k) !} S(n-k, k), \\
\mathrm{B}_{n, k}(0,0,3,4, \ldots, n-k+1) & =\frac{n !}{(n-k) !} S(n-k, k ; 1), \\
\mathrm{B}_{n, k}(x, 2,3,4, \ldots, n-k+1) & =\left(\begin{array}{c}
n \\
k
\end{array}\right) \sum_{r=0}^{k}\left(\begin{array}{c}
k \\
r
\end{array}\right)(k-r)^{n-k}(x-1)^{r}, \\
\mathrm{~B}_{n, k}(0,1,2,3, \ldots, n-k) & =\sum_{r=0}^{n}(-1)^{n-r}\left(\begin{array}{c}
n \\
r
\end{array}\right) k^{r} S(n-r, k ; 1),
\end{aligned}
$$


where $S(n, k ; r)$ for $n \geq k \geq 0$ and $r \geq 0$ are known as the $r$-associate Stirling numbers of the second kind and were defined by

$$
\left(e^{x}-\sum_{i=0}^{r} \frac{x^{i}}{i !}\right)^{k}=\left(\sum_{j=r+1}^{\infty} \frac{x^{j}}{j !}\right)^{k}=k ! \sum_{n=(r+1) k}^{\infty} S(n, k ; r) \frac{x^{n}}{n !} .
$$

in [21, p. 303, eq. (1.2)]. The formula 1.18 gives a closed formula for the special case $a_{1}=1, a_{2}=2$, and $\alpha=0$ in 1.19 . Substituting $1-\alpha$ for $x$ in (1.17) leads to

$$
\begin{aligned}
& \mathrm{B}_{n, k}(1-\alpha, 2-\alpha, 3-\alpha, \ldots, n-k+1-\alpha) \\
&=k^{n-k}\left(\begin{array}{l}
n \\
k
\end{array}\right) \sum_{\ell=0}^{k}(-1)^{\ell}\left(\begin{array}{c}
k \\
\ell
\end{array}\right)\left[\sum_{q=0}^{n-k} \frac{(-1)^{q}}{k^{q}}\left(\begin{array}{c}
n-k \\
q
\end{array}\right) \frac{S(q+\ell, \ell)}{\left(\begin{array}{c}
q+\ell \\
\ell
\end{array}\right)}\right] \alpha^{\ell} .
\end{aligned}
$$

Letting $x=0$ in (1.17) or taking $\alpha=1$ in 1.24 results in

$$
\mathrm{B}_{n, k}(0,1,2,3, \ldots, n-k)=k^{n-k}\left(\begin{array}{l}
n \\
k
\end{array}\right) \sum_{\ell=0}^{k}(-1)^{\ell}\left(\begin{array}{l}
k \\
\ell
\end{array}\right) \sum_{q=0}^{n-k} \frac{(-1)^{q}}{k^{q}}\left(\begin{array}{c}
n-k \\
q
\end{array}\right) \frac{S(q+\ell, \ell)}{\left(\begin{array}{c}
q+\ell \\
\ell
\end{array}\right)}
$$

which, due to useless of associate Stirling numbers of the second kind, is more elementary than (1.23). In [78, Section 2], an alternative expression for (1.21) was derived in term of the Stirling numbers of the second kind $S(n, k)$.

1.13. Howard's numbers. In the paper [20] and closely related references therein, the following numbers and their generating functions were considered:

$$
\begin{aligned}
\frac{x^{2}}{2} \frac{1}{e^{x}-x-1}=\sum_{n=0}^{\infty} A_{n} \frac{x_{n}}{n !}, & \frac{x}{e^{x}-1}=\sum_{n=0}^{\infty} B_{n} \frac{x^{n}}{n !}, \\
\frac{2 x}{e^{x}+1}=\sum_{n=1}^{\infty} G_{n} \frac{x^{n}}{n !}, & -\frac{x^{2}}{(x-2)\left(e^{x}-x-1\right)}=\sum_{n=0}^{\infty} A_{n}^{\prime} \frac{x^{n}}{n !}, \\
-\frac{2 x}{(x-2)\left(e^{x}-1\right)}=\sum_{n=0}^{\infty} B_{n}^{\prime} \frac{x^{n}}{n !}, & -\frac{4}{(x-2)\left(e^{x}+1\right)}=\sum_{n=0}^{\infty} G_{n}^{\prime} \frac{x^{n}}{n !}, \\
\frac{x^{3}}{6} \frac{1}{x\left(e^{x}+1\right)-2\left(e^{x}-1\right)}=\sum_{n=0}^{\infty} V_{n} \frac{x^{n}}{n !}, & -\frac{4}{x\left(e^{x}-1\right)-2\left(e^{x}+1\right)}=\sum_{n=0}^{\infty} W_{n} \frac{x^{n}}{n !},
\end{aligned}
$$

and

$$
\begin{aligned}
\left(1-\frac{x}{2}\right)\left[x\left(e^{x}+1\right)-2\left(e^{x}-1\right)\right]^{k} & =k ! \sum_{n=3 k}^{\infty} A(n, k) \frac{x^{n}}{n !}, \\
{\left[x\left(e^{x}+1\right)-2\left(e^{x}-1\right)\right]^{k} } & =k ! \sum_{n=3 k}^{\infty} V(n, k) \frac{x^{n}}{n !}, \\
{\left[(x-2)\left(e^{x}-1\right)\right]^{k} } & =k ! \sum_{n=k}^{\infty} W(n, k) \frac{x^{n}}{n !} .
\end{aligned}
$$

Among them, the numbers $B_{n}, G_{n}$, and $V_{n}$ are called the Bernoulli, Genocchi, and van der Pol numbers respectively. In 20, among other things, the closed formulas and identities

$$
\begin{aligned}
W_{n} & =\sum_{k=1}^{n} \sum_{r=0}^{n}(-1)^{k+r} \frac{k ! r !}{2^{k+r}}\left(\begin{array}{l}
n \\
r
\end{array}\right)\left(\begin{array}{l}
k \\
r
\end{array}\right) S(n-r, k), \\
V(n, k) & =\mathrm{B}_{n, k}(0,0,1,2, \ldots, n-k-1),
\end{aligned}
$$




$$
\begin{aligned}
W(n, k) & =\mathrm{B}_{n, k}(-2,0,1,2,3, \ldots, n-k-1) \\
& =\sum_{r=0}^{n} r !\left(\begin{array}{l}
n \\
r
\end{array}\right)\left(\begin{array}{l}
k \\
r
\end{array}\right)(-2)^{k-r} S(n-r, k)
\end{aligned}
$$

were obtained.

\section{Closed formulas for some numbers Discussed By Howard}

In this section, we will discover or recover some relations and closed formulas for those numbers $A_{n}, B_{n}, G_{n}, A_{n}^{\prime}, B_{n}^{\prime}, G_{n}^{\prime}, V_{n}, W_{n}, A(n, k), V(n, k)$, and $W(n, k)$ which have been discussed in [20].

Theorem 2.1. For $n \geq k \geq 0$, we have

$$
\begin{aligned}
\mathrm{B}_{n, k}\left(\frac{1}{2 \cdot 3}, \frac{1}{3 \cdot 4}, \ldots, \frac{1}{(n-k+2)(n-k+3)}\right) \\
=\frac{(-1)^{k}}{[2(n+k)] ! !}\left(\begin{array}{l}
n \\
k
\end{array}\right) \sum_{m=0}^{n-k} m !\langle-2 k\rangle_{n-k-m}\left(\begin{array}{c}
n-k \\
m
\end{array}\right)\left(\begin{array}{c}
n+k \\
m
\end{array}\right) \\
\quad \times \sum_{\ell=0}^{k}(-1)^{\ell}\left(\begin{array}{c}
k \\
\ell
\end{array}\right) \sum_{q=0}^{n+k} 2^{q}\langle k-\ell\rangle_{n+k-q}\left(\begin{array}{c}
n+k \\
q
\end{array}\right) \frac{S(\ell+q, \ell)}{\left(\begin{array}{c}
\ell+q \\
\ell
\end{array}\right)}
\end{aligned}
$$

and

$$
\begin{aligned}
A_{n}=\frac{1}{2^{n} n !} \sum_{k=0}^{n} \frac{\left(\begin{array}{c}
n \\
k
\end{array}\right)}{\left(\begin{array}{c}
n+k \\
k
\end{array}\right)} \sum_{m=0}^{n-k} m !\langle-2 k\rangle_{n-k-m}\left(\begin{array}{c}
n-k \\
m
\end{array}\right)\left(\begin{array}{c}
n+k \\
m
\end{array}\right) \\
\quad \times \sum_{\ell=0}^{k}(-1)^{\ell}\left(\begin{array}{c}
k \\
\ell
\end{array}\right) \sum_{q=0}^{n+k} 2^{q}\langle k-\ell\rangle_{n+k-q}\left(\begin{array}{c}
n+k \\
q
\end{array}\right) \frac{S(\ell+q, \ell)}{\left(\begin{array}{c}
\ell+q \\
\ell
\end{array}\right)} .
\end{aligned}
$$

Proof. Let $u=u(x)=\frac{2\left(e^{x}-x-1\right)}{x^{2}}$. It is easy to see that

$$
\lim _{x \rightarrow 0} \frac{1}{u(x)}=\lim _{x \rightarrow 0}\left(\frac{x^{2}}{2} \frac{1}{e^{x}-x-1}\right)=1
$$

and

$$
\begin{gathered}
\lim _{x \rightarrow 0} u^{(n)}(x)=\lim _{x \rightarrow 0}\left[\frac{2\left(e^{x}-x-1\right)}{x^{2}}\right]^{(n)}=\lim _{x \rightarrow 0}\left(\frac{2}{x^{2}} \sum_{k=2}^{\infty} \frac{x^{k}}{k !}\right)^{(n)} \\
=2 \lim _{x \rightarrow 0}\left[\sum_{k=0}^{\infty} \frac{x^{k}}{(k+2) !}\right]^{(n)}=2 \lim _{x \rightarrow 0} \sum_{k=n}^{\infty} \frac{\langle k\rangle_{n}}{(k+2) !} x^{k-n} \\
=2 \frac{\langle n\rangle_{n}}{(n+2) !}=2 \frac{n !}{(n+2) !}=\frac{2}{(n+1)(n+2)} .
\end{gathered}
$$

Therefore, by virtue of the Faà di Bruno formula $(1.1)$, we obtain

$$
\begin{gathered}
A_{n}=\lim _{x \rightarrow 0}\left[\frac{1}{u(x)}\right]^{(n)}=\lim _{x \rightarrow 0} \sum_{k=0}^{n}\left(\frac{1}{u}\right)^{(k)} \mathrm{B}_{n, k}\left(u^{\prime}(x), u^{\prime \prime}(x), \ldots, u^{(n-k+1)}(x)\right) \\
=\lim _{x \rightarrow 0} \sum_{k=0}^{n} \frac{(-1)^{k} k !}{u^{k+1}} \mathrm{~B}_{n, k}\left(u^{\prime}(x), u^{\prime \prime}(x), \ldots, u^{(n-k+1)}(x)\right) \\
=\sum_{k=0}^{n}(-1)^{k} k ! 2^{k} \mathrm{~B}_{n, k}\left(\frac{1}{2 \cdot 3}, \frac{1}{3 \cdot 4}, \ldots, \frac{1}{(n-k+2)(n-k+3)}\right) .
\end{gathered}
$$


In [7, p. 133], it was listed that

$$
\frac{1}{k !}\left(\sum_{m=1}^{\infty} x_{m} \frac{t^{m}}{m !}\right)^{k}=\sum_{n=k}^{\infty} \mathrm{B}_{n, k}\left(x_{1}, x_{2}, \ldots, x_{n-k+1}\right) \frac{t^{n}}{n !}
$$

for $k \geq 0$. From 2.3 it can be deduced that

$$
\begin{aligned}
& \sum_{n=k}^{\infty} \mathrm{B}_{n, k}\left(\frac{1}{2 \cdot 3}, \frac{1}{3 \cdot 4}, \ldots, \frac{1}{(n-k+2)(n-k+3)}\right) \frac{t^{n}}{n !} \\
= & \frac{1}{k !}\left[\sum_{m=1}^{\infty} \frac{1}{(m+1)(m+2)} \frac{t^{m}}{m !}\right]^{k}=\frac{1}{k !}\left[\sum_{m=1}^{\infty} \frac{t^{m}}{(m+2) !}\right]^{k} \\
= & \frac{1}{k !}\left(\frac{e^{t}-1-t-\frac{t^{2}}{2}}{t^{2}}\right)^{k}=\frac{t^{k}}{k !}\left(\frac{e^{t}-1-t-\frac{t^{2}}{2}}{t^{3}}\right)^{k}
\end{aligned}
$$

which is equivalent to

$$
\begin{gathered}
\sum_{n=0}^{\infty} \mathrm{B}_{n+k, k}\left(\frac{1}{2 \cdot 3}, \frac{1}{3 \cdot 4}, \ldots, \frac{1}{(n+2)(n+3)}\right) \frac{k !}{(n+k) !} t^{n}=\left(\frac{e^{t}-1-t-\frac{t^{2}}{2}}{t^{3}}\right)^{k} \\
=\frac{1}{t^{2 k}}\left[\frac{e^{t}-1}{t}-\left(1+\frac{t}{2}\right)\right]^{k}=\frac{1}{t^{2 k}} \sum_{\ell=0}^{k}(-1)^{k-\ell}\left(\begin{array}{c}
k \\
\ell
\end{array}\right)\left(\frac{e^{t}-1}{t}\right)^{\ell}\left(1+\frac{t}{2}\right)^{k-\ell} .
\end{gathered}
$$

This implies that

$$
\begin{gathered}
\mathrm{B}_{n+k, k}\left(\frac{1}{2 \cdot 3}, \frac{1}{3 \cdot 4}, \ldots, \frac{1}{(n+2)(n+3)}\right) \frac{n ! k !}{(n+k) !} \\
=\lim _{t \rightarrow 0}\left[\frac{1}{t^{2 k}} \sum_{\ell=0}^{k}(-1)^{k-\ell}\left(\begin{array}{c}
k \\
\ell
\end{array}\right)\left(\frac{e^{t}-1}{t}\right)^{\ell}\left(1+\frac{t}{2}\right)^{k-\ell}\right]^{(n)} \\
=\lim _{t \rightarrow 0} \sum_{m=0}^{n}\left(\begin{array}{c}
n \\
m
\end{array}\right)\left(\frac{1}{t^{2 k}}\right)^{(m)}\left[\sum_{\ell=0}^{k}(-1)^{k-\ell}\left(\begin{array}{c}
k \\
\ell
\end{array}\right)\left(\frac{e^{t}-1}{t}\right)^{\ell}\left(1+\frac{t}{2}\right)^{k-\ell}\right]^{(n-m)} \\
=\lim _{t \rightarrow 0} \sum_{m=0}^{n}\left(\begin{array}{c}
n \\
m
\end{array}\right) \frac{\langle-2 k\rangle_{m}}{t^{2 k+m}} \sum_{\ell=0}^{k}(-1)^{k-\ell}\left(\begin{array}{c}
k \\
\ell
\end{array}\right)\left[\left(\frac{e^{t}-1}{t}\right)^{\ell}\left(1+\frac{t}{2}\right)^{k-\ell}\right]^{(n-m)} \\
=\lim _{t \rightarrow 0} \frac{1}{t^{2 k+n}} \sum_{m=0}^{n}\left(\begin{array}{c}
n \\
m
\end{array}\right)\langle-2 k\rangle_{m} \sum_{\ell=0}^{k}(-1)^{k-\ell}\left(\begin{array}{l}
k \\
\ell
\end{array}\right) t^{n-m} \\
\times\left[\left(\frac{e^{t}-1}{t}\right)^{\ell}\left(1+\frac{t}{2}\right)^{k-\ell}\right]^{(n-m)} \\
=\frac{1}{(2 k+n) !} \lim _{t \rightarrow 0}\left(\sum_{m=0}^{n}\langle-2 k\rangle_{n-m}\left(\begin{array}{c}
n \\
m
\end{array}\right) \sum_{\ell=0}^{k}(-1)^{k-\ell}\left(\begin{array}{l}
k \\
\ell
\end{array}\right) t^{m}\right. \\
\left.\times\left[\left(\frac{e^{t}-1}{t}\right)^{\ell}\left(1+\frac{t}{2}\right)^{k-\ell}\right]^{(m)}\right)^{(2 k+n)} \\
=\frac{(-1)^{k}}{(2 k+n) !} \lim _{t \rightarrow 0} \sum_{m=0}^{n}\langle-2 k\rangle_{n-m}\left(\begin{array}{c}
n \\
m
\end{array}\right) \sum_{\ell=0}^{k}(-1)^{\ell}\left(\begin{array}{l}
k \\
\ell
\end{array}\right) \\
\times \sum_{q=0}^{2 k+n}\left(\begin{array}{c}
2 k+n \\
q
\end{array}\right)\left(t^{m}\right)^{(q)}\left[\left(\frac{e^{t}-1}{t}\right)^{\ell}\left(1+\frac{t}{2}\right)^{k-\ell}\right]^{(m+2 k+n-q)}
\end{gathered}
$$




$$
\begin{aligned}
& =\frac{(-1)^{k}}{(2 k+n) !} \sum_{m=0}^{n}\langle-2 k\rangle_{n-m}\left(\begin{array}{c}
n \\
m
\end{array}\right) \sum_{\ell=0}^{k}(-1)^{\ell}\left(\begin{array}{c}
k \\
\ell
\end{array}\right)\left(\begin{array}{c}
2 k+n \\
m
\end{array}\right) \\
& \times m ! \lim _{t \rightarrow 0}\left[\left(\frac{e^{t}-1}{t}\right)^{\ell}\left(1+\frac{t}{2}\right)^{k-\ell}\right]^{(2 k+n)} \\
& =\frac{(-1)^{k}}{(2 k+n) !} \sum_{m=0}^{n} m !\langle-2 k\rangle_{n-m}\left(\begin{array}{c}
n \\
m
\end{array}\right)\left(\begin{array}{c}
2 k+n \\
m
\end{array}\right) \sum_{\ell=0}^{k}(-1)^{\ell}\left(\begin{array}{c}
k \\
\ell
\end{array}\right) \\
& \times \lim _{t \rightarrow 0} \sum_{q=0}^{2 k+n}\left(\begin{array}{c}
2 k+n \\
q
\end{array}\right)\left[\left(\frac{e^{t}-1}{t}\right)^{\ell}\right]^{(q)}\left[\left(1+\frac{t}{2}\right)^{k-\ell}\right]^{(2 k+n-q)} \\
& =\frac{(-1)^{k}}{(2 k+n) !} \sum_{m=0}^{n} m !\langle-2 k\rangle_{n-m}\left(\begin{array}{c}
n \\
m
\end{array}\right)\left(\begin{array}{c}
2 k+n \\
m
\end{array}\right) \sum_{\ell=0}^{k}(-1)^{\ell}\left(\begin{array}{c}
k \\
\ell
\end{array}\right) \\
& \times \lim _{t \rightarrow 0} \sum_{q=0}^{2 k+n}\left(\begin{array}{c}
2 k+n \\
q
\end{array}\right)\left[\ell ! \sum_{p=q}^{\infty} \frac{S(p+\ell, \ell)}{(p+\ell) !}\langle p\rangle_{q} t^{p-q}\right] \\
& \times \frac{\langle k-\ell\rangle_{2 k+n-q}}{2^{2 k+n-q}}\left(1+\frac{t}{2}\right)^{k-\ell-(2 k+n-q)} \\
& =\frac{(-1)^{k}}{(2 k+n) !} \sum_{m=0}^{n} m !\langle-2 k\rangle_{n-m}\left(\begin{array}{c}
n \\
m
\end{array}\right)\left(\begin{array}{c}
2 k+n \\
m
\end{array}\right) \sum_{\ell=0}^{k}(-1)^{\ell}\left(\begin{array}{l}
k \\
\ell
\end{array}\right) \\
& \times \sum_{q=0}^{2 k+n}\left(\begin{array}{c}
2 k+n \\
q
\end{array}\right)\left[\ell ! \frac{S(q+\ell, \ell)}{(q+\ell) !}\langle q\rangle_{q}\right] \frac{\langle k-\ell\rangle_{2 k+n-q}}{2^{2 k+n-q}},
\end{aligned}
$$

where we used the L'Hôspital rule and the generating function

$$
\frac{\left(e^{x}-1\right)^{k}}{k !}=\sum_{n=k}^{\infty} S(n, k) \frac{x^{n}}{n !}
$$

for the Stirling numbers of the second kind $S(n, k)$. As a result, we procure

$$
\begin{aligned}
& \mathrm{B}_{n+k, k}\left(\frac{1}{2 \cdot 3}, \frac{1}{3 \cdot 4}, \ldots, \frac{1}{(n+2)(n+3)}\right) \\
&=\frac{(-1)^{k}}{[2(n+2 k)] ! !}\left(\begin{array}{c}
n+k \\
k
\end{array}\right) \sum_{m=0}^{n} m !\langle-2 k\rangle_{n-m}\left(\begin{array}{c}
n \\
m
\end{array}\right)\left(\begin{array}{c}
n+2 k \\
m
\end{array}\right) \\
& \quad \times \sum_{\ell=0}^{k}(-1)^{\ell}\left(\begin{array}{c}
k \\
\ell
\end{array}\right) \sum_{q=0}^{n+2 k} 2^{q}\langle k-\ell\rangle_{n+2 k-q}\left(\begin{array}{c}
n+2 k \\
q
\end{array}\right) \frac{S(q+\ell, \ell)}{\left(\begin{array}{c}
q+\ell \\
\ell
\end{array}\right)}
\end{aligned}
$$

which is equivalent to (2.1). From (2.1), we derive $(2.2)$. The closed formula $(2.2)$ was numerically verified to be true by the software Mathematica. The proof of Theorem 2.1 is thus complete.

Theorem 2.2 ([14, Theorem 1]). For $n \geq 0$, we have

$$
B_{n}=\sum_{i=0}^{n}(-1)^{i} \frac{\left(\begin{array}{c}
n+1 \\
i+1
\end{array}\right)}{\left(\begin{array}{c}
n+i \\
i
\end{array}\right)} S(n+i, i) .
$$

Proof. Let $v=v(x)=\frac{e^{x}-1}{x}$. Then

$$
\lim _{x \rightarrow 0}[v(x)]^{(n)}=\lim _{x \rightarrow 0}\left(\frac{e^{x}-1}{x}\right)^{(n)}=\lim _{x \rightarrow 0}\left(\sum_{k=1}^{\infty} \frac{x^{k-1}}{k !}\right)^{(n)}
$$




$$
=\lim _{x \rightarrow 0}\left[\sum_{k=0}^{\infty} \frac{x^{k}}{(k+1) !}\right]^{(n)}=\lim _{x \rightarrow 0} \sum_{k=n}^{\infty} \frac{\langle k\rangle_{n}}{(k+1) !} x^{k-n}=\frac{\langle n\rangle_{n}}{(n+1) !}=\frac{1}{n+1}
$$

and, in light of 1.1 and 1.16 in sequence,

$$
\begin{gathered}
B_{n}=\lim _{x \rightarrow 0}\left(\frac{x}{e^{x}-1}\right)^{(n)}=\lim _{x \rightarrow 0}\left[\frac{1}{v(x)}\right]^{(n)} \\
=\lim _{x \rightarrow 0} \sum_{k=0}^{n}\left(\frac{1}{v}\right)^{(k)} \mathrm{B}_{n, k}\left(v^{\prime}(x), v^{\prime \prime}(x), \ldots, v^{(n-k+1)}(x)\right) \\
=\sum_{k=0}^{n} \lim _{x \rightarrow 0} \frac{(-1)^{k} k !}{v^{k+1}} \lim _{x \rightarrow 0} \mathrm{~B}_{n, k}\left(v^{\prime}(x), v^{\prime \prime}(x), \ldots, v^{(n-k+1)}(x)\right) \\
=\sum_{k=0}^{n}(-1)^{k} k ! \mathrm{B}_{n, k}\left(\frac{1}{2}, \frac{1}{3}, \ldots, \frac{1}{n-k+2}\right) \\
=\sum_{k=0}^{n} \frac{1}{\left(\begin{array}{c}
n+k \\
n
\end{array}\right)} \sum_{\ell=0}^{k}(-1)^{\ell}\left(\begin{array}{c}
n+k \\
k-\ell
\end{array}\right) S(n+\ell, \ell)=\sum_{i=0}^{n}(-1)^{i} \frac{\left(\begin{array}{c}
n+1 \\
i+1
\end{array}\right)}{\left(\begin{array}{c}
n+i \\
i
\end{array}\right)} S(n+i, i) .
\end{gathered}
$$

The proof of Theorem 2.2 is complete.

Theorem 2.3 ([11, Theorem 1.1]). For $n \in \mathbb{N}$, we have

$$
G_{n}=n \sum_{k=0}^{n-1}(-1)^{k} \frac{k !}{2^{k}} S(n-1, k) .
$$

Proof. The generating function of the Genocchi numbers $G_{n}$ can be rewritten as

$$
\frac{2}{e^{x}+1}=\sum_{n=1}^{\infty} G_{n} \frac{x^{n-1}}{n !}=\sum_{n=0}^{\infty} \frac{G_{n+1}}{n+1} \frac{x^{n}}{n !}
$$

which means that, when denoting $w=w(x)=e^{x}+1$,

$$
\begin{gathered}
\frac{G_{n+1}}{n+1}=\lim _{x \rightarrow 0}\left(\frac{2}{e^{x}+1}\right)^{(n)}=2 \lim _{x \rightarrow 0} \sum_{k=0}^{n}\left(\frac{1}{w}\right)^{(k)} \mathrm{B}_{n, k}\left(e^{x}, e^{x}, \ldots, e^{x}\right) \\
=2 \lim _{x \rightarrow 0} \sum_{k=0}^{n} \frac{(-1)^{k} k !}{w^{k+1}} e^{k x} \mathrm{~B}_{n, k}(1,1, \ldots, 1) \\
=\sum_{k=0}^{n}(-1)^{k} \frac{k !}{2^{k}} \mathrm{~B}_{n, k}(1,1, \ldots, 1)=\sum_{k=0}^{n}(-1)^{k} \frac{k !}{2^{k}} S(n, k)
\end{gathered}
$$

which gives

$$
G_{n+1}=(n+1) \sum_{k=0}^{n}(-1)^{k} \frac{k !}{2^{k}} S(n, k), \quad n \geq 0
$$

and then recovers [11, Theorem 1.1]. The proof of Theorem 2.3 is complete.

Theorem 2.4. For $n \geq 0$, we have

$$
A_{n+1}^{\prime}-\frac{n+1}{2} A_{n}^{\prime}=A_{n+1}
$$

and

$$
A_{n}^{\prime}=\sum_{k=0}^{n} \frac{\langle n\rangle_{k}}{2^{k}} A_{n-k}=\sum_{p=0}^{n} \frac{\langle n\rangle_{n-p}}{2^{n-p}} A_{p}
$$




$$
\begin{array}{r}
=\frac{1}{2^{n}} \sum_{p=0}^{n} \frac{\langle n\rangle_{n-p}}{p !} \sum_{k=0}^{p} \frac{\left(\begin{array}{c}
p \\
k
\end{array}\right)}{\left(\begin{array}{c}
p+k \\
k
\end{array}\right)} \sum_{m=0}^{p-k} m !\langle-2 k\rangle_{p-k-m}\left(\begin{array}{c}
p-k \\
m
\end{array}\right)\left(\begin{array}{c}
p+k \\
m
\end{array}\right) \\
\times \sum_{\ell=0}^{k}(-1)^{\ell}\left(\begin{array}{c}
k \\
\ell
\end{array}\right) \sum_{q=0}^{p+k} 2^{q}\langle k-\ell\rangle_{p+k-q}\left(\begin{array}{c}
p+k \\
q
\end{array}\right) \frac{S(q+\ell, \ell)}{\left(\begin{array}{c}
q+\ell \\
\ell
\end{array}\right)} .
\end{array}
$$

Proof. The generating function of the numbers $A_{n}^{\prime}$ can be rearranged as

$$
\begin{aligned}
& \frac{x^{2}}{2\left(e^{x}-x-1\right)}=\left(1-\frac{x}{2}\right) \sum_{n=0}^{\infty} A_{n}^{\prime} \frac{x^{n}}{n !}=\sum_{n=0}^{\infty} A_{n}^{\prime} \frac{x^{n}}{n !}-\frac{1}{2} \sum_{n=0}^{\infty} A_{n}^{\prime} \frac{x^{n+1}}{n !} \\
& =\sum_{n=0}^{\infty} A_{n}^{\prime} \frac{x^{n}}{n !}-\frac{1}{2} \sum_{n=1}^{\infty} A_{n-1}^{\prime} \frac{x^{n}}{(n-1) !}=A_{0}^{\prime}+\sum_{n=1}^{\infty}\left(A_{n}^{\prime}-\frac{n}{2} A_{n-1}^{\prime}\right) \frac{x^{n}}{n !}
\end{aligned}
$$

which implies that $A_{0}^{\prime}=1$ and

$$
A_{n}^{\prime}-\frac{n}{2} A_{n-1}^{\prime}=\lim _{x \rightarrow 0}\left[\frac{x^{2}}{2\left(e^{x}-x-1\right)}\right]^{(n)}=A_{n}
$$

for $n \in \mathbb{N}$. Recursing consecutively and making use of 2.1, the formula 2.6 follows. The proof of Theorem 2.4 is complete.

Theorem 2.5. For $n \geq 0$, we have

$$
B_{0}^{\prime}=1, \quad B_{n+1}^{\prime}-\frac{n+1}{2} B_{n}^{\prime}=B_{n+1},
$$

and

$$
B_{n}^{\prime}=\sum_{p=0}^{n} \frac{\langle n\rangle_{n-p}}{2^{n-p}} \sum_{i=0}^{p}(-1)^{i} \frac{\left(\begin{array}{c}
p+1 \\
i+1
\end{array}\right)}{\left(\begin{array}{c}
p+i \\
i
\end{array}\right)} S(p+i, i) .
$$

Proof. The generating function of the numbers $B_{n}^{\prime}$ can be formulated as

$$
\frac{x}{e^{x}-1}=\left(1-\frac{x}{2}\right) \sum_{n=0}^{\infty} B_{n}^{\prime} \frac{x^{n}}{n !}=B_{0}^{\prime}+\sum_{n=1}^{\infty}\left(B_{n}^{\prime}-\frac{n}{2} B_{n-1}^{\prime}\right) \frac{x^{n}}{n !}
$$

which means that the relation 2.7$)$ is true. Recursing and utilizing 2.5$)$, we obtain

$$
B_{n}^{\prime}=\sum_{k=0}^{n} \frac{\langle n\rangle_{k}}{2^{k}} B_{n-k}=\sum_{p=0}^{n} \frac{\langle n\rangle_{n-p}}{2^{n-p}} B_{p}=\sum_{p=0}^{n} \frac{\langle n\rangle_{n-p}}{2^{n-p}} \sum_{i=0}^{p}(-1)^{i} \frac{\left(\begin{array}{c}
p+1 \\
i+1
\end{array}\right)}{\left(\begin{array}{c}
p+i \\
i
\end{array}\right)} S(p+i, i) .
$$

The proof of Theorem 2.5 is complete.

Theorem 2.6. For $n \geq 0$, we have

$$
G_{0}^{\prime}=1, \quad G_{n+1}^{\prime}-\frac{n+1}{2} G_{n}^{\prime}=\frac{G_{n+2}}{n+2},
$$

and

$$
G_{n}^{\prime}=\sum_{\ell=0}^{n} \frac{\langle n\rangle_{n-\ell}}{2^{n-\ell}} \sum_{k=0}^{\ell}(-1)^{k} \frac{k !}{2^{k}} S(\ell, k) .
$$

Proof. It is easy to see that

$$
\begin{aligned}
\frac{2 x}{e^{x}+1}=x\left(1-\frac{x}{2}\right)\left[-\frac{4}{(x-2)\left(e^{x}+1\right)}\right] & =x\left(1-\frac{x}{2}\right) \sum_{n=0}^{\infty} G_{n}^{\prime} \frac{x^{n}}{n !} \\
= & G_{0}^{\prime} x+\sum_{n=2}^{\infty} n\left(G_{n-1}^{\prime}-\frac{n-1}{2} G_{n-2}^{\prime}\right) \frac{x^{n}}{n !}
\end{aligned}
$$


which leads to $G_{0}^{\prime}=G_{1}=1$ and

$$
G_{n-1}^{\prime}-\frac{n-1}{2} G_{n-2}^{\prime}=\frac{G_{n}}{n}, \quad n \geq 2 .
$$

Recursively, it follows that

$$
G_{n}^{\prime}=\sum_{k=0}^{n} \frac{\langle n\rangle_{k}}{2^{k}} \frac{G_{n-k+1}}{n-k+1}=\sum_{\ell=0}^{n} \frac{\langle n\rangle_{n-\ell}}{2^{n-\ell}} \frac{G_{\ell+1}}{\ell+1}=\sum_{\ell=0}^{n} \frac{\langle n\rangle_{n-\ell}}{2^{n-\ell}} \sum_{k=0}^{\ell}(-1)^{k} \frac{k !}{2^{k}} S(\ell, k) .
$$

The proof of Theorem 2.6 is complete.

Theorem 2.7. For $n \geq k \geq 0$, we have

$$
\begin{aligned}
& \mathrm{B}_{n, k}\left(\frac{1}{3 \cdot 4}, \frac{1}{4 \cdot 5}, \ldots, \frac{1}{(n-k+3)(n-k+4)}\right)=\frac{(-1)^{k}}{(n+2 k) ! 6^{k}}\left(\begin{array}{l}
n \\
k
\end{array}\right) \\
\times & \sum_{q=0}^{n-k}\langle-3 k\rangle_{q}(n-k-q) !\left(\begin{array}{c}
n-k \\
q
\end{array}\right)\left(\begin{array}{c}
n+2 k \\
n-k-q
\end{array}\right) \sum_{\ell=0}^{k} 12^{\ell}\left(\begin{array}{c}
k \\
\ell
\end{array}\right) \sum_{p=0}^{n+2 k}(n+2 k-p) ! \\
\times & \left(\begin{array}{c}
n+2 k \\
p
\end{array}\right) \frac{S(p+\ell, \ell)}{\left(\begin{array}{c}
p+\ell \\
\ell
\end{array}\right)} \sum_{s=0}^{k-\ell}\left(\begin{array}{c}
k-\ell \\
s
\end{array}\right) \frac{(-6)^{s}}{(n-p+2 \ell+2 s) !} \sum_{\beta=1}^{s}\left(\begin{array}{c}
s \\
\beta
\end{array}\right) \beta^{n-p+2 \ell+2 s}
\end{aligned}
$$

and

$$
\begin{aligned}
V_{n}=\sum_{k=0}^{n} \frac{k !}{(n+2 k) !}\left(\begin{array}{l}
n \\
k
\end{array}\right) \sum_{q=0}^{n-k}\langle-3 k\rangle_{q}(n-k-q) !\left(\begin{array}{c}
n-k \\
q
\end{array}\right)\left(\begin{array}{c}
n+2 k \\
n-k-q
\end{array}\right) \\
\quad \times \sum_{\ell=0}^{k} 12^{\ell}\left(\begin{array}{l}
k \\
\ell
\end{array}\right) \sum_{p=0}^{n+2 k}(n+2 k-p) !\left(\begin{array}{c}
n+2 k \\
p
\end{array}\right) \frac{S(p+\ell, \ell)}{\left(\begin{array}{c}
p+\ell \\
\ell
\end{array}\right)} \\
\quad \times \sum_{s=0}^{k-\ell}\left(\begin{array}{c}
k-\ell \\
s
\end{array}\right) \frac{(-6)^{s}}{(n-p+2 \ell+2 s) !} \sum_{\beta=1}^{s}\left(\begin{array}{c}
s \\
\beta
\end{array}\right) \beta^{n-p+2 \ell+2 s} .
\end{aligned}
$$

Proof. The generating function of the numbers $V_{n}$ can be rewritten as

$$
\left[\frac{x\left(e^{x}+1\right)-2\left(e^{x}-1\right)}{x^{3} / 6}\right]^{-1}=\sum_{n=0}^{\infty} V_{n} \frac{x^{n}}{n !} .
$$

It is easy to see that

$$
\begin{aligned}
& \frac{x\left(e^{x}+1\right)-2\left(e^{x}-1\right)}{x^{3} / 6}=\frac{6}{x^{3}}\left[x+\sum_{k=0}^{\infty} \frac{x^{k+1}}{k !}-2 \sum_{k=1}^{\infty} \frac{x^{k}}{k !}\right] \\
= & \frac{6}{x^{3}}\left[x+\sum_{k=1}^{\infty} \frac{x^{k}}{(k-1) !}-2 \sum_{k=1}^{\infty} \frac{x^{k}}{k !}\right]=\frac{6}{x^{3}} \sum_{k=2}^{\infty}\left[\frac{1}{(k-1) !}-\frac{2}{k !}\right] x^{k} \\
= & \frac{6}{x^{3}} \sum_{k=3}^{\infty}(k-2) \frac{x^{k}}{k !}=6 \sum_{k=3}^{\infty}(k-2) \frac{x^{k-3}}{k !}=6 \sum_{k=0}^{\infty}(k+1) \frac{x^{k}}{(k+3) !}
\end{aligned}
$$

and that

$$
\begin{aligned}
& \lim _{x \rightarrow 0}\left[\frac{x\left(e^{x}+1\right)-2\left(e^{x}-1\right)}{x^{3} / 6}\right]^{(n)}=6 \lim _{x \rightarrow 0}\left[\sum_{k=0}^{\infty}(k+1) \frac{x^{k}}{(k+3) !}\right]^{(n)} \\
= & 6 \lim _{x \rightarrow 0} \sum_{k=n}^{\infty}(k+1) \frac{\langle k\rangle_{n} x^{k-n}}{(k+3) !}=6(n+1) \frac{\langle n\rangle_{n}}{(n+3) !}=\frac{6}{(n+2)(n+3)} .
\end{aligned}
$$


Accordingly, by similar arguments as above, it follows that

$$
\begin{aligned}
V_{n} & =\lim _{x \rightarrow 0} \frac{\mathrm{d}^{n}}{\mathrm{~d} x^{n}}\left[\frac{x\left(e^{x}+1\right)-2\left(e^{x}-1\right)}{x^{3} / 6}\right]^{-1} \\
& =\sum_{k=0}^{n}(-1)^{k} k ! 6^{k} \mathrm{~B}_{n, k}\left(\frac{1}{3 \cdot 4}, \frac{1}{4 \cdot 5}, \ldots, \frac{1}{(n-k+3)(n-k+4)}\right) .
\end{aligned}
$$

By virtue of the formula 2.3 , we have

$$
\begin{aligned}
& \sum_{n=k}^{\infty} \mathrm{B}_{n, k}\left(\frac{1}{3 \cdot 4}, \frac{1}{4 \cdot 5}, \ldots, \frac{1}{(n-k+3)(n-k+4)}\right) \frac{t^{n}}{n !} \\
= & \frac{1}{k !}\left[\sum_{m=1}^{\infty} \frac{1}{(m+2)(m+3)} \frac{t^{m}}{m !}\right]^{k} \\
= & \frac{1}{k !}\left[\frac{6 t\left(e^{t}+1\right)-t^{3}-12\left(e^{t}-1\right)}{6 t^{3}}\right]^{k} \\
= & \frac{1}{k !} \frac{2^{k}}{t^{2 k}}\left(\frac{e^{t}+1}{2}-\frac{t^{2}}{12}-\frac{e^{t}-1}{t}\right)^{k} \\
= & \frac{1}{k !} \frac{2^{k}}{t^{2 k}} \sum_{\ell=0}^{k}(-1)^{\ell}\left(\begin{array}{c}
k \\
\ell
\end{array}\right)\left(\frac{e^{t}+1}{2}-\frac{t^{2}}{12}\right)^{k-\ell}\left(\frac{e^{t}-1}{t}\right)^{\ell}
\end{aligned}
$$

which is equivalent to

$$
\begin{aligned}
\sum_{\ell=0}^{\infty} \mathrm{B}_{\ell+k, k}\left(\frac{1}{3 \cdot 4}, \frac{1}{4 \cdot 5}, \ldots,\right. & \left.\frac{1}{(\ell+3)(\ell+4)}\right) \frac{1}{\left(\begin{array}{c}
\ell+k \\
k
\end{array}\right)} \frac{t^{\ell}}{\ell !} \\
& =\frac{2^{k}}{t^{3 k}} \sum_{\ell=0}^{k}(-1)^{\ell}\left(\begin{array}{c}
k \\
\ell
\end{array}\right)\left(\frac{e^{t}+1}{2}-\frac{t^{2}}{12}\right)^{k-\ell}\left(\frac{e^{t}-1}{t}\right)^{\ell} .
\end{aligned}
$$

This implies that, by virtue of the L'Hôspital rule and the generating function (2.4) for the Stirling numbers of the second kind $S(n, k)$,

$$
\begin{aligned}
& \frac{1}{\left(\begin{array}{c}
m+k \\
k
\end{array}\right)} \mathrm{B}_{m+k, k}\left(\frac{1}{3 \cdot 4}, \frac{1}{4 \cdot 5}, \ldots, \frac{1}{(m+3)(m+4)}\right) \\
= & \lim _{t \rightarrow 0}\left[\frac{2^{k}}{t^{3 k}} \sum_{\ell=0}^{k}(-1)^{\ell}\left(\begin{array}{c}
k \\
\ell
\end{array}\right)\left(\frac{e^{t}+1}{2}-\frac{t^{2}}{12}\right)^{k-\ell}\left(\frac{e^{t}-1}{t}\right)^{\ell}\right]^{(m)} \\
= & 2^{k} \lim _{t \rightarrow 0} \sum_{q=0}^{m}\left(\begin{array}{c}
m \\
q
\end{array}\right)\left(\frac{1}{t^{3 k}}\right)^{(q)}\left[\sum_{\ell=0}^{k}(-1)^{\ell}\left(\begin{array}{c}
k \\
\ell
\end{array}\right)\left(\frac{e^{t}+1}{2}-\frac{t^{2}}{12}\right)^{k-\ell}\left(\frac{e^{t}-1}{t}\right)^{\ell}\right]^{(m-q)} \\
= & 2^{k} \lim _{t \rightarrow 0} \sum_{q=0}^{m}\left(\begin{array}{c}
m \\
q
\end{array}\right) \frac{\langle-3 k\rangle_{q}}{t^{3 k+q}} \sum_{\ell=0}^{k}(-1)^{\ell}\left(\begin{array}{c}
k \\
\ell
\end{array}\right)\left[\left(\frac{e^{t}+1}{2}-\frac{t^{2}}{12}\right)^{k-\ell}\left(\frac{e^{t}-1}{t}\right)^{\ell}\right]^{(m-q)} \\
= & 2^{k} \lim _{t \rightarrow 0} \frac{1}{t^{3 k+m}} \sum_{q=0}^{m}\left(\begin{array}{c}
m \\
q
\end{array}\right)\langle-3 k\rangle_{q} \sum_{\ell=0}^{k}(-1)^{\ell}\left(\begin{array}{c}
k \\
\ell
\end{array}\right) t^{m-q} \\
& \times\left[\left(\frac{e^{t}+1}{2}-\frac{t^{2}}{12}\right)^{k-\ell}\left(\frac{e^{t}-1}{t}\right)^{\ell}\right]^{(m-q)} \\
= & \frac{2^{k}}{(3 k+m) !} \lim _{t \rightarrow 0} \sum_{q=0}^{m}\left(\begin{array}{c}
m \\
q
\end{array}\right)\langle-3 k\rangle_{q} \sum_{\ell=0}^{k}(-1)^{\ell}\left(\begin{array}{l}
k \\
\ell
\end{array}\right)
\end{aligned}
$$




$$
\begin{aligned}
& \times\left(t^{m-q}\left[\left(\frac{e^{t}+1}{2}-\frac{t^{2}}{12}\right)^{k-\ell}\left(\frac{e^{t}-1}{t}\right)^{\ell}\right]^{(m-q)}\right)^{(3 k+m)} \\
& =\frac{2^{k}}{(3 k+m) !} \lim _{t \rightarrow 0} \sum_{q=0}^{m}\left(\begin{array}{c}
m \\
q
\end{array}\right)\langle-3 k\rangle_{q} \sum_{\ell=0}^{k}(-1)^{\ell}\left(\begin{array}{c}
k \\
\ell
\end{array}\right) \sum_{p=0}^{3 k+m}\left(\begin{array}{c}
3 k+m \\
p
\end{array}\right) \\
& \times\left(t^{m-q}\right)^{(p)}\left(\left[\left(\frac{e^{t}+1}{2}-\frac{t^{2}}{12}\right)^{k-\ell}\left(\frac{e^{t}-1}{t}\right)^{\ell}\right]^{(m-q)}\right)^{(3 k+m-p)} \\
& =\frac{2^{k}}{(3 k+m) !} \sum_{q=0}^{m}\left(\begin{array}{c}
m \\
q
\end{array}\right)\langle-3 k\rangle_{q} \sum_{\ell=0}^{k}(-1)^{\ell}\left(\begin{array}{c}
k \\
\ell
\end{array}\right)\left(\begin{array}{c}
3 k+m \\
m-q
\end{array}\right)(m-q) \text { ! } \\
& \times \lim _{t \rightarrow 0}\left[\left(\frac{e^{t}+1}{2}-\frac{t^{2}}{12}\right)^{k-\ell}\left(\frac{e^{t}-1}{t}\right)^{\ell}\right]^{(3 k+m)} \\
& =\frac{2^{k}}{(3 k+m) !} \sum_{q=0}^{m}\left(\begin{array}{c}
m \\
q
\end{array}\right)\langle-3 k\rangle_{q} \sum_{\ell=0}^{k}(-1)^{\ell}\left(\begin{array}{c}
k \\
\ell
\end{array}\right)\left(\begin{array}{c}
3 k+m \\
m-q
\end{array}\right)(m-q) \text { ! } \\
& \times \lim _{t \rightarrow 0} \sum_{p=0}^{3 k+m}\left(\begin{array}{c}
3 k+m \\
p
\end{array}\right)\left[\left(\frac{e^{t}+1}{2}-\frac{t^{2}}{12}\right)^{k-\ell}\right]^{(3 k+m-p)}\left[\left(\frac{e^{t}-1}{t}\right)^{\ell}\right]^{(p)} \\
& =\frac{2^{k}}{(3 k+m) !} \sum_{q=0}^{m}\left(\begin{array}{c}
m \\
q
\end{array}\right)\langle-3 k\rangle_{q} \sum_{\ell=0}^{k}(-1)^{\ell}\left(\begin{array}{c}
k \\
\ell
\end{array}\right)\left(\begin{array}{c}
3 k+m \\
m-q
\end{array}\right)(m-q) ! \\
& \times \lim _{t \rightarrow 0} \sum_{p=0}^{3 k+m}\left(\begin{array}{c}
3 k+m \\
p
\end{array}\right)\left[\left(\frac{e^{t}+1}{2}-\frac{t^{2}}{12}\right)^{k-\ell}\right]^{(3 k+m-p)} \ell ! \sum_{r=p}^{\infty} \frac{S(r+\ell, \ell)}{(r+\ell) !}\langle r\rangle_{p} t^{r-p} \\
& =\frac{2^{k}}{(3 k+m) !} \sum_{q=0}^{m}\left(\begin{array}{c}
m \\
q
\end{array}\right)\langle-3 k\rangle_{q} \sum_{\ell=0}^{k}(-1)^{\ell}\left(\begin{array}{c}
k \\
\ell
\end{array}\right)\left(\begin{array}{c}
3 k+m \\
m-q
\end{array}\right)(m-q) \text { ! } \\
& \times \sum_{p=0}^{3 k+m}\left(\begin{array}{c}
3 k+m \\
p
\end{array}\right) \lim _{t \rightarrow 0}\left[\left(\frac{e^{t}+1}{2}-\frac{t^{2}}{12}\right)^{k-\ell}\right]^{(3 k+m-p)} \frac{S(p+\ell, \ell)}{\left(\begin{array}{c}
p+\ell \\
\ell
\end{array}\right)},
\end{aligned}
$$

where

$$
\begin{aligned}
& \lim _{t \rightarrow 0}\left[\left(\frac{e^{t}+1}{2}-\frac{t^{2}}{12}\right)^{k-\ell}\right]^{(\tau)} \\
= & \lim _{t \rightarrow 0}\left[\sum_{s=0}^{k-\ell}(-1)^{k-\ell-s}\left(\begin{array}{c}
k-\ell \\
s
\end{array}\right)\left(\frac{e^{t}+1}{2}\right)^{s}\left(\frac{t^{2}}{12}\right)^{k-\ell-s}\right]^{(\tau)} \\
= & \lim _{t \rightarrow 0} \sum_{s=0}^{k-\ell} \frac{(-1)^{k-\ell-s}}{12^{k-\ell-s}}\left(\begin{array}{c}
k-\ell \\
s
\end{array}\right)\left[\left(\frac{e^{t}+1}{2}\right)^{s} t^{2(k-\ell-s)}\right]^{(\tau)} \\
= & \lim _{t \rightarrow 0} \sum_{s=0}^{k-\ell} \frac{(-1)^{k-\ell-s}}{12^{k-\ell-s} 2^{s}}\left(\begin{array}{c}
k-\ell \\
s
\end{array}\right)\left[t^{2(k-\ell-s)} \sum_{\beta=0}^{s}\left(\begin{array}{c}
s \\
\beta
\end{array}\right) e^{\beta t}\right]^{(\tau)} \\
= & \lim _{t \rightarrow 0} \sum_{s=0}^{k-\ell} \frac{(-1)^{k-\ell-s}}{12^{k-\ell-s} 2^{s}}\left(\begin{array}{c}
k-\ell \\
s
\end{array}\right)\left[t^{2(k-\ell-s)} \sum_{\beta=0}^{s}\left(\begin{array}{c}
s \\
\beta
\end{array}\right) \sum_{\gamma=0}^{\infty} \frac{\beta^{\gamma} t^{\gamma}}{\gamma !}\right]^{(\tau)} \\
= & \lim _{t \rightarrow 0} \sum_{s=0}^{k-\ell} \frac{(-1)^{k-\ell-s}}{12^{k-\ell-s} 2^{s}}\left(\begin{array}{c}
k-\ell \\
s
\end{array}\right)\left[\sum_{\beta=0}^{s}\left(\begin{array}{c}
s \\
\beta
\end{array}\right) \sum_{\gamma=0}^{\infty} \frac{\beta^{\gamma}}{\gamma !} t^{2(k-\ell-s)+\gamma}\right]^{(\tau)}
\end{aligned}
$$




$$
\begin{aligned}
& =\lim _{t \rightarrow 0} \sum_{s=0}^{k-\ell} \frac{(-1)^{k-\ell-s}}{12^{k-\ell-s} 2^{s}}\left(\begin{array}{c}
k-\ell \\
s
\end{array}\right) \sum_{\beta=0}^{s}\left(\begin{array}{l}
s \\
\beta
\end{array}\right) \sum_{\gamma=0}^{\infty} \frac{\beta^{\gamma}}{\gamma !}\langle 2(k-\ell-s)+\gamma\rangle_{\tau} t^{2(k-\ell-s)+\gamma-\tau} \\
& =\sum_{s=0}^{k-\ell} \frac{(-1)^{k-\ell-s}}{12^{k-\ell-s} 2^{s}}\left(\begin{array}{c}
k-\ell \\
s
\end{array}\right) \sum_{\beta=0}^{s}\left(\begin{array}{l}
s \\
\beta
\end{array}\right) \frac{\beta^{\tau-2(k-\ell-s)}}{[\tau-2(k-\ell-s)] !}\langle\tau\rangle_{\tau} .
\end{aligned}
$$

Consequently, we have

$$
\begin{aligned}
& \mathrm{B}_{m+k, k}\left(\frac{1}{3 \cdot 4}, \frac{1}{4 \cdot 5}, \ldots, \frac{1}{(m+3)(m+4)}\right) \\
= & \frac{(-1)^{k}}{(3 k+m) ! 6^{k}}\left(\begin{array}{c}
m+k \\
k
\end{array}\right) \sum_{q=0}^{m}\langle-3 k\rangle_{q}\left(\begin{array}{c}
m \\
q
\end{array}\right)\left(\begin{array}{c}
3 k+m \\
m-q
\end{array}\right)(m-q) ! \\
& \times \sum_{\ell=0}^{k} 12^{\ell}\left(\begin{array}{c}
k \\
\ell
\end{array}\right) \sum_{p=0}^{3 k+m}(3 k+m-p) !\left(\begin{array}{c}
3 k+m \\
p
\end{array}\right) \frac{S(p+\ell, \ell)}{\left(\begin{array}{c}
p+\ell \\
\ell
\end{array}\right)} \\
& \times \sum_{s=0}^{k-\ell}\left(\begin{array}{c}
k-\ell \\
s
\end{array}\right) \frac{(-6)^{s}}{(k+m-p+2 \ell+2 s) !} \sum_{\beta=1}^{s}\left(\begin{array}{c}
s \\
\beta
\end{array}\right) \beta^{k+m-p+2 \ell+2 s} .
\end{aligned}
$$

The proof of Theorem 2.7 is complete.

Theorem 2.8. For $n \geq k \geq 0$, we have

$$
\begin{aligned}
W(n, k) & =\mathrm{B}_{n, k}(-2,0,1,2, \ldots, n-k-1) \\
& =\left(\begin{array}{l}
n \\
k
\end{array}\right) \sum_{\ell=0}^{n-k}(-2)^{\ell}\langle k\rangle_{n-k-\ell}\left(\begin{array}{c}
n-k \\
\ell
\end{array}\right) \frac{S(\ell+k, k)}{\left(\begin{array}{c}
\ell+k \\
k
\end{array}\right)}
\end{aligned}
$$

and

$$
W_{n}=\sum_{k=0}^{n} \frac{k !}{4^{k}} W(n, k)=\sum_{k=0}^{n} \frac{k !}{4^{k}}\left(\begin{array}{l}
n \\
k
\end{array}\right) \sum_{\ell=0}^{n-k}(-2)^{\ell}\langle k\rangle_{n-k-\ell}\left(\begin{array}{c}
n-k \\
\ell
\end{array}\right) \frac{S(\ell+k, k)}{\left(\begin{array}{c}
\ell+k \\
k
\end{array}\right)} .
$$

Proof. It is not difficult to find that

$$
\begin{gathered}
x\left(e^{x}-1\right)-2\left(e^{x}+1\right)=\sum_{k=1}^{\infty} \frac{x^{k+1}}{k !}-2 \sum_{k=0}^{\infty} \frac{x^{k}}{k !}-2 \\
=\sum_{k=2}^{\infty} \frac{x^{k}}{(k-1) !}-2 \sum_{k=2}^{\infty} \frac{x^{k}}{k !}-2 x-4=\sum_{k=3}^{\infty}(k-2) \frac{x^{k}}{k !}-2 x-4 .
\end{gathered}
$$

Consequently, it follows that

$$
\left.\left[x\left(e^{x}-1\right)-2\left(e^{x}+1\right)\right]^{\prime}\right|_{x=0}=-2,\left.\quad\left[x\left(e^{x}-1\right)-2\left(e^{x}+1\right)\right]^{\prime \prime}\right|_{x=0}=0,
$$

and

$$
\left.\left[x\left(e^{x}-1\right)-2\left(e^{x}+1\right)\right]^{(n)}\right|_{x=0}=n-2, \quad n \geq 3 .
$$

Therefore, we can derive that

$$
\begin{gathered}
W_{n}=\lim _{x \rightarrow 0} \frac{\mathrm{d}^{n}}{\mathrm{~d} x^{n}}\left[-\frac{4}{x\left(e^{x}-1\right)-2\left(e^{x}+1\right)}\right] \\
=-4 \sum_{k=0}^{n}(-1)^{k} \frac{k !}{(-4)^{k+1}} \mathrm{~B}_{n, k}(-2,0,1,2, \ldots, n-k-1) \\
=\sum_{k=0}^{n} \frac{k !}{4^{k}} \mathrm{~B}_{n, k}(-2,0,1,2, \ldots, n-k-1)
\end{gathered}
$$


for $n \geq 0$. Since

$$
\begin{aligned}
& \sum_{n=k}^{\infty} W(n, k) \frac{x^{n}}{n !}=\frac{1}{k !}\left[(x-2)\left(e^{x}-1\right)\right]^{k}=\frac{1}{k !}\left[(x-2) \sum_{n=1}^{\infty} \frac{x^{n}}{n !}\right]^{k} \\
& =\frac{1}{k !}\left[\sum_{n=2}^{\infty} \frac{x^{n}}{(n-1) !}-2 \sum_{n=1}^{\infty} \frac{x^{n}}{n !}\right]^{k}=\frac{1}{k !}\left[-2 x+\sum_{n=2}^{\infty}(n-2) \frac{x^{n}}{n !}\right]^{k},
\end{aligned}
$$

comparing with 2.3 recovers the first equality in (1.27). Consequently, we have

$$
\begin{gathered}
\frac{W(n+k, k)}{\left(\begin{array}{c}
n+k \\
k
\end{array}\right)}=\frac{\mathrm{B}_{n+k, k}(-2,0,1,2, \ldots, n-1)}{\left(\begin{array}{c}
n+k \\
k
\end{array}\right)} \\
=\lim _{t \rightarrow 0} \frac{\mathrm{d}^{n}}{\mathrm{~d} t^{n}}\left[(t-2)\left(\frac{e^{t}-1}{t}\right)\right]^{k}=\lim _{t \rightarrow 0} \sum_{\ell=0}^{n}\left(\begin{array}{c}
n \\
\ell
\end{array}\right)\left[(t-2)^{k}\right]^{(\ell)}\left[\left(\frac{e^{t}-1}{t}\right)^{k}\right]^{(n-\ell)} \\
=\lim _{t \rightarrow 0} \sum_{\ell=0}^{n}\left(\begin{array}{c}
n \\
\ell
\end{array}\right)\langle k\rangle_{\ell}(t-2)^{k-\ell}\left[k ! \sum_{p=n-\ell}^{\infty} \frac{S(p+k, k)}{(p+k) !}\langle p\rangle_{n-\ell} t^{p-(n-\ell)}\right] \\
=\sum_{\ell=0}^{n}\left(\begin{array}{c}
n \\
\ell
\end{array}\right)\langle k\rangle_{\ell}(-2)^{k-\ell} k ! \frac{S(n-\ell+k, k)}{(n-\ell+k) !}\langle n-\ell\rangle_{n-\ell} \\
=k ! \sum_{\ell=0}^{n}\left(\begin{array}{l}
n \\
\ell
\end{array}\right)\langle k\rangle_{n-\ell}(-2)^{\ell} \frac{S(\ell+k, k)}{(\ell+k) !}\langle\ell\rangle_{\ell} .
\end{gathered}
$$

As a result, it follows that

$$
\mathrm{B}_{n+k, k}(-2,0,1,2, \ldots, n-1)=\left(\begin{array}{c}
n+k \\
k
\end{array}\right) \sum_{\ell=0}^{n}(-2)^{\ell}\langle k\rangle_{n-\ell}\left(\begin{array}{c}
n \\
\ell
\end{array}\right) \frac{S(\ell+k, k)}{\left(\begin{array}{c}
\ell+k \\
k
\end{array}\right)} .
$$

The proof of Theorem 2.8 is complete.

Theorem 2.9. For $n \geq 3 k$, we have

$$
A(3 k, k)=V(3 k, k), \quad A(n+1, k)=V(n+1, k)-\frac{n+1}{2} V(n, k)
$$

and

$$
\begin{aligned}
V(n, k)=\mathrm{B}_{n, k}(0,0 & 1,2, \ldots, n-k-1)=2^{k}\left(\begin{array}{l}
n \\
k
\end{array}\right) \sum_{\ell=0}^{k}(-1)^{k-\ell}\left(\begin{array}{c}
k \\
\ell
\end{array}\right) \\
& \times \sum_{q=0}^{n-k}\left(\begin{array}{c}
n-k \\
q
\end{array}\right) \frac{S(n-q-\ell, k-\ell)}{\left(\begin{array}{c}
n-q-\ell \\
n-q
\end{array}\right)} \sum_{m=0}^{q} \frac{\langle\ell\rangle_{m}}{2^{m}} S(q, m) .
\end{aligned}
$$

Proof. Since

$$
\begin{gathered}
k ! \sum_{n=3 k}^{\infty} A(n, k) \frac{x^{n}}{n !}=\left(1-\frac{x}{2}\right)\left[x\left(e^{x}+1\right)-2\left(e^{x}-1\right)\right]^{k} \\
=\left(1-\frac{x}{2}\right) k ! \sum_{n=3 k}^{\infty} V(n, k) \frac{x^{n}}{n !}=k !\left[\sum_{n=3 k}^{\infty} V(n, k) \frac{x^{n}}{n !}-\frac{1}{2} \sum_{n=3 k}^{\infty} V(n, k) \frac{x^{n+1}}{n !}\right] \\
=k !\left[\sum_{n=3 k}^{\infty} V(n, k) \frac{x^{n}}{n !}-\frac{1}{2} \sum_{n=3 k+1}^{\infty} V(n-1, k) \frac{x^{n}}{(n-1) !}\right] \\
=k ! V(3 k, k)+k ! \sum_{n=3 k+1}^{\infty}\left[V(n, k)-\frac{n}{2} V(n-1, k)\right] \frac{x^{n}}{n !},
\end{gathered}
$$

then the relations in 2.10 follow immediately. 
Combining the formula 2.3 with

$$
\sum_{n=3 k}^{\infty} V(n, k) \frac{x^{n}}{n !}=\frac{1}{k !}\left[x\left(e^{x}+1\right)-2\left(e^{x}-1\right)\right]^{k}=\frac{1}{k !}\left[\sum_{\ell=3}^{\infty}(\ell-2) \frac{x^{\ell}}{\ell !}\right]^{k}
$$

reveals that the formula 1.26 for $n \geq 3 k$. By virtue of 2.3$)$, we have

$$
\begin{aligned}
& \sum_{n=k}^{\infty} \mathrm{B}_{n, k}(0,0,1,2, \ldots, n-k-1) \frac{t^{n}}{n !}=\frac{1}{k !}\left[\sum_{m=3}^{\infty}(m-2) \frac{t^{m}}{m !}\right]^{k}, \\
& \sum_{n=0}^{\infty} \frac{\mathrm{B}_{n+k, k}(0,0,1,2, \ldots, n-1)}{\left(\begin{array}{c}
n+k \\
k
\end{array}\right)} \frac{t^{n}}{n !}=2^{k}\left(\frac{e^{t}+1}{2}-\frac{e^{t}-1}{t}\right)^{k}, \\
& \frac{\mathrm{B}_{n+k, k}(0,0,1,2, \ldots, n-1)}{\left(\begin{array}{c}
n+k \\
k
\end{array}\right)}=2^{k} \lim _{t \rightarrow 0} \frac{\mathrm{d}^{n}}{\mathrm{~d} t^{n}}\left(\frac{e^{t}+1}{2}-\frac{e^{t}-1}{t}\right)^{k} \\
& =2^{k} \lim _{t \rightarrow 0} \frac{\mathrm{d}^{n}}{\mathrm{~d} t^{n}} \sum_{\ell=0}^{k}(-1)^{k-\ell}\left(\begin{array}{l}
k \\
\ell
\end{array}\right)\left(\frac{e^{t}+1}{2}\right)^{\ell}\left(\frac{e^{t}-1}{t}\right)^{k-\ell} \\
& =2^{k} \lim _{t \rightarrow 0} \sum_{\ell=0}^{k}(-1)^{k-\ell}\left(\begin{array}{l}
k \\
\ell
\end{array}\right) \sum_{q=0}^{n}\left(\begin{array}{l}
n \\
q
\end{array}\right)\left[\left(\frac{e^{t}+1}{2}\right)^{\ell}\right]^{(q)}\left[\left(\frac{e^{t}-1}{t}\right)^{k-\ell}\right]^{(n-q)} \\
& =\lim _{t \rightarrow 0} \sum_{\ell=0}^{k}(-2)^{k-\ell}\left(\begin{array}{l}
k \\
\ell
\end{array}\right) \sum_{q=0}^{n}\left(\begin{array}{l}
n \\
q
\end{array}\right) \sum_{m=0}^{q}\langle\ell\rangle_{m}\left(e^{t}+1\right)^{\ell-m} \mathrm{~B}_{q, m}\left(e^{t}, e^{t}, \ldots, e^{t}\right) \\
& \times\left[(k-\ell) ! \sum_{p=n-q}^{\infty} \frac{S(p+k-\ell, k-\ell)}{(p+k-\ell) !}\langle p\rangle_{n-q} t^{p-(n-q)}\right] \\
& =\sum_{\ell=0}^{k}(-2)^{k-\ell}\left(\begin{array}{l}
k \\
\ell
\end{array}\right) \sum_{q=0}^{n}\left(\begin{array}{l}
n \\
q
\end{array}\right) \sum_{m=0}^{q}\langle\ell\rangle_{m} 2^{\ell-m} \mathrm{~B}_{q, m}(1,1, \ldots, 1) \\
& \times(k-\ell) ! \frac{S(n-q+k-\ell, k-\ell)}{(n-q+k-\ell) !}\langle n-q\rangle_{n-q} \\
& =(-2)^{k} \sum_{\ell=0}^{k}(-1)^{\ell}\left(\begin{array}{l}
k \\
\ell
\end{array}\right) \sum_{q=0}^{n}\left(\begin{array}{l}
n \\
q
\end{array}\right) \frac{S(n-q+k-\ell, k-\ell)}{\left(\begin{array}{c}
n-q+k-\ell \\
n-q
\end{array}\right)} \sum_{m=0}^{q} \frac{\langle\ell\rangle_{m}}{2^{m}} S(q, m) \text {. }
\end{aligned}
$$

The formula 2.11 follows readily. The proof of Theorem 2.9 is complete.

\section{A Closed Formula For a special Bell polynomial}

The quantity $\mathrm{B}_{n, k}\left(a_{1}, a_{2}, 3-\alpha, 4-\alpha, \ldots,(n-k+1)-\alpha\right)$ was specially investigated in [20], but there was no closed formula developed for it there. We now start out to derive a closed and explicit formula for $\mathrm{B}_{n, k}\left(a_{1}, a_{2}, 3-\alpha, 4-\alpha, \ldots,(n-k+1)-\alpha\right)$.

Theorem 3.1. For $n \geq k \geq 0$, we have

$$
\begin{aligned}
\mathrm{B}_{n, k}\left(a_{1}, a_{2}, 3\right. & -\alpha, 4-\alpha, \ldots,(n-k+1)-\alpha) \\
=\frac{n !}{k !} \sum_{m=0}^{k}\left(\begin{array}{c}
k \\
m
\end{array}\right) \sum_{p+q+r=n-k}(-\alpha)^{m-q}\left(a_{1}+\alpha\right)^{k-m-p} & \\
& \times\left(\frac{a_{2}+\alpha}{2}-1\right)^{p} \frac{\langle k-m\rangle_{p}\langle m\rangle_{q}}{p ! q ! r !} \frac{S(r+m, m)}{\left(\begin{array}{c}
r+m \\
r
\end{array}\right)} .
\end{aligned}
$$


Proof. By virtue of 2.3 , we have

$$
\begin{gathered}
\sum_{n=k}^{\infty} \mathrm{B}_{n, k}\left(a_{1}, a_{2}, 3-\alpha, 4-\alpha, \ldots,(n-k+1)-\alpha\right) \frac{t^{n}}{n !} \\
=\frac{1}{k !}\left(a_{1} t+\frac{a_{2}}{2} t^{2}+\sum_{m=3}^{\infty}(m-\alpha) \frac{t^{m}}{m !}\right)^{k} \\
=\frac{t^{k}}{k !}\left[a_{1}+\alpha+\left(\frac{a_{2}+\alpha}{2}-1\right) t+(t-\alpha)\left(\frac{e^{t}-1}{t}\right)\right]^{k}
\end{gathered}
$$

which is equivalent to

$$
\begin{gathered}
\sum_{n=0}^{\infty} \frac{\mathrm{B}_{n+k, k}\left(a_{1}, a_{2}, 3-\alpha, 4-\alpha, \ldots,(n+1)-\alpha\right)}{\left(\begin{array}{c}
n+k \\
k
\end{array}\right)} \frac{t^{n}}{n !} \\
=\left[a_{1}+\alpha+\left(\frac{a_{2}}{2}+\frac{\alpha}{2}-1\right) t+(t-\alpha)\left(\frac{e^{t}-1}{t}\right)\right]^{k} .
\end{gathered}
$$

Therefore, it follows that, when $A=a_{1}+\alpha$ and $B=\frac{a_{2}+\alpha}{2}-1$,

$$
\begin{aligned}
& \mathrm{B}_{n+k, k}\left(a_{1}, a_{2}, 3-\alpha, 4-\alpha, \ldots,(n+1)-\alpha\right) \\
& =\left(\begin{array}{c}
n+k \\
k
\end{array}\right) \lim _{t \rightarrow 0} \frac{\mathrm{d}^{n}}{\mathrm{~d} t^{n}}\left[a_{1}+\alpha+\left(\frac{a_{2}}{2}+\frac{\alpha}{2}-1\right) t+(t-\alpha)\left(\frac{e^{t}-1}{t}\right)\right]^{k} \\
& =\left(\begin{array}{c}
n+k \\
k
\end{array}\right) \lim _{t \rightarrow 0} \frac{\mathrm{d}^{n}}{\mathrm{~d} t^{n}} \sum_{\ell=0}^{k}\left(\begin{array}{l}
k \\
\ell
\end{array}\right)(A+B t)^{\ell}(t-\alpha)^{k-\ell}\left(\frac{e^{t}-1}{t}\right)^{k-\ell} \\
& =\left(\begin{array}{c}
n+k \\
k
\end{array}\right) \lim _{t \rightarrow 0} \sum_{\ell=0}^{k}\left(\begin{array}{l}
k \\
\ell
\end{array}\right) \sum_{p+q+r=n} \frac{n !}{p ! q ! r !}\left[(A+B t)^{\ell}\right]^{(p)} \\
& \times\left[(t-\alpha)^{k-\ell}\right]^{(q)}\left[\left(\frac{e^{t}-1}{t}\right)^{k-\ell}\right]^{(r)} \\
& =\left(\begin{array}{c}
n+k \\
k
\end{array}\right) \lim _{t \rightarrow 0} \sum_{\ell=0}^{k}\left(\begin{array}{l}
k \\
\ell
\end{array}\right) \sum_{p+q+r=n} \frac{n !}{p ! q ! r !}\langle\ell\rangle_{p} B^{p}(A+B t)^{\ell-p}\langle k-\ell\rangle_{q}(t-\alpha)^{k-\ell-q} \\
& \times(k-\ell) ! \sum_{s=r}^{\infty} \frac{S(s+k-\ell, k-\ell)}{(s+k-\ell) !}\langle s\rangle_{r} t^{s-r} \\
& =\left(\begin{array}{c}
n+k \\
k
\end{array}\right) \sum_{\ell=0}^{k}\left(\begin{array}{l}
k \\
\ell
\end{array}\right) \sum_{p+q+r=n} \frac{n !}{p ! q ! r !}\langle\ell\rangle_{p} B^{p} A^{\ell-p}\langle k-\ell\rangle_{q} \\
& \times(-\alpha)^{k-\ell-q}(k-\ell) ! \frac{S(r+k-\ell, k-\ell)}{(r+k-\ell) !}\langle r\rangle_{r} \\
& =n !\left(\begin{array}{c}
n+k \\
k
\end{array}\right) \sum_{\ell=0}^{k}\left(\begin{array}{l}
k \\
\ell
\end{array}\right) \sum_{p+q+r=n} \frac{(-\alpha)^{k-\ell-q} B^{p} A^{\ell-p}\langle\ell\rangle_{p}\langle k-\ell\rangle_{q}}{p ! q ! r !} \frac{S(r+k-\ell, k-\ell)}{\left(\begin{array}{c}
r+k-\ell \\
r
\end{array}\right)} .
\end{aligned}
$$

The equality 3.1 is thus proved. The proof of Theorem 3.1 is thus complete.

\section{REMARKS AND COMPARISONS}

We now list several remarks and comparisons about our main results and some known results. 
Remark 4.1. Although those formulas in Theorem 2.7 are a little complex, but they are surely closed formulas for

$$
\mathrm{B}_{n, k}\left(\frac{1}{3 \cdot 4}, \frac{1}{4 \cdot 5}, \ldots, \frac{1}{(n-k+3)(n-k+4)}\right)
$$

and $V_{n}$. If computing along alternative approaches the $n$th derivative of the function

$$
\frac{1}{t^{2 k}}\left(\frac{e^{t}+1}{2}-\frac{t^{2}}{12}-\frac{e^{t}-1}{t}\right)^{k}
$$

with respect to $t$, then we can find alternative closed formulas for the quantity 4.1 and the sequence $V_{n}$.

Remark 4.2. The formulas 2.8 and 2.9 are slightly different from the formulas 1.27 and 1.25 respectively.

Remark 4.3. Taking $a_{1}=x, a_{2}=1+x$, and $\alpha=1-x$ in Theorem 3.1 gives

$$
\begin{aligned}
\mathrm{B}_{n, k}(x, 1+x, 2+x, 3+x, \ldots, & n-k+x) \\
= & \frac{n !}{k !} \sum_{\ell=0}^{k}\left(\begin{array}{l}
k \\
\ell
\end{array}\right) \sum_{q+r=n-k} \frac{\langle\ell\rangle_{q}}{q ! r !} \frac{S(r+\ell, \ell)}{\left(\begin{array}{c}
r+\ell \\
r
\end{array}\right)}(x-1)^{\ell-q} .
\end{aligned}
$$

Comparing this with 1.17 demonstrates that

$$
\begin{aligned}
& \frac{k^{n-k}}{(n-k) !} \sum_{\ell=0}^{k}\left(\begin{array}{l}
k \\
\ell
\end{array}\right)\left[\sum_{q=0}^{n-k} \frac{(-1)^{q}}{k^{q}}\left(\begin{array}{c}
n-k \\
q
\end{array}\right) \frac{S(q+\ell, \ell)}{\left(\begin{array}{c}
q+\ell \\
\ell
\end{array}\right)}\right](x-1)^{\ell} \\
& =\sum_{\ell=0}^{k}\left(\begin{array}{l}
k \\
\ell
\end{array}\right) \sum_{m=0}^{n-k} \frac{\langle\ell\rangle_{n-k-m}}{(n-k-m) ! m !} \frac{S(m+\ell, \ell)}{\left(\begin{array}{c}
m+\ell \\
m
\end{array}\right)}(x-1)^{\ell+m-(n-k)} \\
& =\sum_{\ell=0}^{k}\left(\begin{array}{l}
k \\
\ell
\end{array}\right) \sum_{m=n-k-\ell}^{n-k} \frac{\langle\ell\rangle_{n-k-m}}{(n-k-m) ! m !} \frac{S(m+\ell, \ell)}{\left(\begin{array}{c}
m+\ell \\
m
\end{array}\right)}(x-1)^{\ell+m-(n-k)} \\
& =\sum_{\ell=0}^{k}\left(\begin{array}{l}
k \\
\ell
\end{array}\right) \sum_{q=0}^{\ell} \frac{\langle\ell\rangle_{\ell-q}}{(\ell-q) ![(n-k)-(\ell-q)] !} \frac{S(n-k+q, \ell)}{\left(\begin{array}{c}
n-k+q \\
(n-k)-(\ell-q)
\end{array}\right)}(x-1)^{q} \\
& =\sum_{\ell=0}^{k} \sum_{q=0}^{\ell}\left(\begin{array}{l}
k \\
\ell
\end{array}\right)\left(\begin{array}{l}
\ell \\
q
\end{array}\right) \frac{\ell ! S(n-k+q, \ell)}{(n-k+q) !}(x-1)^{q} \\
& =\sum_{q=0}^{k} \sum_{\ell=q}^{k}\left(\begin{array}{l}
k \\
\ell
\end{array}\right)\left(\begin{array}{l}
\ell \\
q
\end{array}\right) \frac{\ell ! S(n-k+q, \ell)}{(n-k+q) !}(x-1)^{q} \\
& =\sum_{\ell=0}^{k} \sum_{m=\ell}^{k}\left(\begin{array}{c}
k \\
m
\end{array}\right)\left(\begin{array}{c}
m \\
\ell
\end{array}\right) \frac{m ! S(n-k+\ell, m)}{(n-k+\ell) !}(x-1)^{\ell} \\
& =\sum_{\ell=0}^{k} \sum_{q=0}^{k-\ell}\left(\begin{array}{c}
k \\
\ell+q
\end{array}\right)\left(\begin{array}{c}
\ell+q \\
\ell
\end{array}\right) \frac{(\ell+q) ! S(n-k+\ell, \ell+q)}{(n-k+\ell) !}(x-1)^{\ell}
\end{aligned}
$$

which implies

$$
\begin{aligned}
k^{n} \frac{(n+\ell) !}{n !}\left(\begin{array}{l}
k \\
\ell
\end{array}\right) \sum_{q=0}^{n} \frac{(-1)^{q}}{k^{q}}\left(\begin{array}{l}
n \\
q
\end{array}\right) & \frac{S(q+\ell, \ell)}{\left(\begin{array}{c}
q+\ell \\
\ell
\end{array}\right)} \\
& =\sum_{q=0}^{k-\ell}\left(\begin{array}{c}
k \\
\ell+q
\end{array}\right)\left(\begin{array}{c}
\ell+q \\
\ell
\end{array}\right)(\ell+q) ! S(n+\ell, \ell+q) .
\end{aligned}
$$


In particular, letting $\ell=k$ in 4.2 results in an interesting identity

$$
\sum_{q=0}^{n}(-1)^{q}\left(\begin{array}{l}
n \\
q
\end{array}\right) \frac{S(q+k, k)}{k^{q}\left(\begin{array}{c}
q+k \\
k
\end{array}\right)}=\frac{S(n+k, k)}{k^{n}\left(\begin{array}{c}
n+k \\
n
\end{array}\right)} .
$$

Remark 4.4. Letting $a_{1}=1, a_{2}=2$, and $\alpha=0$ in Theorem 3.1 yields

$$
\mathrm{B}_{n, k}(1,2,3,4, \ldots, n-k+1)=\frac{n !}{(n-k) !} \sum_{\ell=0}^{k} \frac{S(n-k, \ell)}{(k-\ell) !} .
$$

Comparing this with 1.18 leads to an identity

$$
\sum_{\ell=0}^{k} \frac{S(n, \ell)}{(k-\ell) !}=\sum_{m=0}^{k} \frac{S(n, k-m)}{m !}=\frac{k^{n}}{k !}
$$

which is a special case of the formula

$$
x^{n}=\sum_{j=0}^{n}\left(\begin{array}{l}
x \\
j
\end{array}\right) j ! S(n, j)
$$

in 99 , p. $116,(9.11)]$

Remark 4.5. Setting $a_{1}=x, a_{2}=2$, and $\alpha \rightarrow 0$ in Theorem 3.1 reduces to

$$
\begin{gathered}
\mathrm{B}_{n, k}(x, 2,3,4, \ldots, n-k+1) \\
k ! \\
\sum_{\ell=0}^{k}\left(\begin{array}{l}
k \\
\ell
\end{array}\right) \sum_{k-\ell+r=n-k} \frac{\langle k-\ell\rangle_{k-\ell}}{(k-\ell) ! r !} x^{\ell} \frac{S(r+k-\ell, k-\ell)}{\left(\begin{array}{c}
r+k-\ell \\
r
\end{array}\right)} \\
=\frac{n !}{k !} \sum_{\ell=0}^{k}\left(\begin{array}{l}
k \\
\ell
\end{array}\right) \frac{1}{(n-2 k+\ell) !} \frac{S(n-k, k-\ell)}{\left(\begin{array}{c}
n-k \\
n-2 k+\ell
\end{array}\right)} x^{\ell} \\
=\left(\begin{array}{l}
n \\
k
\end{array}\right) \sum_{\ell=0}^{k}\left(\begin{array}{l}
k \\
\ell
\end{array}\right)(k-\ell) ! S(n-k, k-\ell) x^{\ell} .
\end{gathered}
$$

Letting $x \rightarrow 0$ in the above equation recovers 1.20 . Comparing 1.22 with the above equation reveals

$$
\sum_{r=0}^{k}\left(\begin{array}{l}
k \\
r
\end{array}\right)(k-r)^{n-k}(x-1)^{r}=\sum_{\ell=0}^{k}\left(\begin{array}{l}
k \\
\ell
\end{array}\right)(k-\ell) ! S(n-k, k-\ell) x^{\ell}
$$

In particular, when $x=1$ in 4.4 , we obtain the equation 4.3 once again.

Remark 4.6. Three special cases

$$
\begin{aligned}
& \mathrm{B}_{n, k}(1,2,3, \ldots, n-k+1)=\left(\begin{array}{l}
n \\
k
\end{array}\right) k^{n-k}, \\
& \mathrm{~B}_{n, k}(0,2,3, \ldots, n-k+1)=\frac{n !}{(n-k) !} S(n-k, k),
\end{aligned}
$$

and

$$
\mathrm{B}_{n, k}(0,0,3,4, \ldots, n-k+1)=n ! \sum_{\ell=0}^{k} \frac{(-1)^{\ell}}{\ell !} \frac{S(n-k-\ell, k-\ell)}{(n-k-\ell) !}
$$

of Theorem 3.1 have been applied in 78 to construct several recursive relations of the Stirling numbers of the second kind $S(n, k)$ which have interpretations in combinatorics and number theory. 
Remark 4.7. In [82, Theorem 1], among other things, the formula

$$
U_{n}(x)=x^{n} \sum_{\ell=0}^{\lfloor n / 2\rfloor}\left(\begin{array}{c}
n+1 \\
2 \ell+1
\end{array}\right)\left(1-\frac{1}{x^{2}}\right)^{\ell}
$$

for the Chebyshev polynomials of the second kind $U_{n}$ was recovered by virtue of the formulas (1.1) and (1.8). We remark that the formula 4.5 was ever recovered in [55. p. 127] simply from a formula

$$
U_{n}(x)=\sum_{m=0}^{\lfloor n / 2\rfloor}\left(\begin{array}{c}
n+1 \\
2 m+1
\end{array}\right) x^{n-2 m}\left(x^{2}-1\right)^{m}
$$

in [24], where $\lfloor x\rfloor$ is the floor function whose value equals the largest integer less than or equal to $x$. However, it seems that the formula (4.6) does not appear in [24].

Remark 4.8. This paper is a slightly corrected and revised version of the electronic preprint 85 .

Funding. The third author was supported by the National Research Foundation of Korea (Grant No. 2018R1D1A1B07041846).

Acknowledgements. The authors are grateful to anonymous referees for their careful corrections and professional comments on the original version of this paper.

\section{REFERENCES}

[1] A. Aboud, J.-P. Bultel, A. Chouria, J.-G. Luque, O. Mallet, Bell polynomials in combinatorial Hopf algebras, arXiv preprint (2014), available online at http://arxiv.org/abs/1402. 2960

[2] M. Abramowitz and I. A. Stegun (Eds), Handbook of Mathematical Functions with Formulas, Graphs, and Mathematical Tables, National Bureau of Standards, Applied Mathematics Series 55, Reprint of the 1972 edition, Dover Publications, Inc., New York, 1992.

[3] P. Appell and J. Kampé de Fériet, Fonctions hypergéométriques et hypersphériques: Polynômes d'Hermite, Gauthier-Villars, Paris, 1926. (French)

[4] A. Z. Broder, The r-Stirling numbers, Discrete Math. 49 (1984), no. 3, 241-259; available online at https://doi.org/10.1016/0012-365X(84)90161-4

[5] L. Carlitz, Weighted Stirling numbers of the first and second kind, I, Fibonacci Quart. 18 (1980), no. 2, 147-162.

[6] C. A. Charalambides, Enumerative Combinatorics, CRC Press Series on Discrete Mathematics and its Applications. Chapman \& Hall/CRC, Boca Raton, FL, 2002.

[7] L. Comtet, Advanced Combinatorics: The Art of Finite and Infinite Expansions, Revised and Enlarged Edition, D. Reidel Publishing Co., 1974; available online at https://doi.org/ 10.1007/978-94-010-2196-8

[8] P. Ferrante, Connections between the iterated (anti)derivatives of $e^{s \sqrt{x}}$ with respect to $x$ and spherical modified Bessel functions of second kind, Georgian Math. J. 21 (2014), no. 4, 421-430; available online at https://doi.org/10.1515/gmj-2014-0041

[9] M. Griffiths and I. Mező, A generalization of Stirling numbers of the second kind via a special multiset, J. Integer Seq. 13 (2010), no. 2, Article 10.2.5, 23 pages.

[10] B.-N. Guo, I. Mező, and F. Qi, An explicit formula for the Bernoulli polynomials in terms of the r-Stirling numbers of the second kind, Rocky Mountain J. Math. 46 (2016), no. 6, 1919-1923; available online at https://doi.org/10.1216/RMJ-2016-46-6-1919

[11] B.-N. Guo and F. Qi, A new explicit formula for the Bernoulli and Genocchi numbers in terms of the Stirling numbers, Glob. J. Math. Anal. 3 (2015), no. 1, 33-36; available online at https://doi.org/10.14419/gjma.v3i1.4168.

[12] B.-N. Guo and F. Qi, Alternative proofs of a formula for Bernoulli numbers in terms of Stirling numbers, Analysis (Berlin) 34 (2014), no. 2, 187-193; available online at https: //doi.org/10.1515/anly-2012-1238

[13] B.-N. Guo and F. Qi, An explicit formula for Bell numbers in terms of Stirling numbers and hypergeometric functions, Glob. J. Math. Anal. 2 (2014), no. 4, 243-248; available online at https://doi.org/10.14419/gjma.v2i4.3310 
[14] B.-N. Guo and F. Qi, An explicit formula for Bernoulli numbers in terms of Stirling numbers of the second kind, J. Anal. Number Theory 3 (2015), no. 1, 27-30; available online at https://doi.org/10.12785/jant/030105.

[15] B.-N. Guo and F. Qi, Explicit formulae for computing Euler polynomials in terms of Stirling numbers of the second kind, J. Comput. Appl. Math. 272 (2014), 251-257; available online at https://doi.org/10.1016/j.cam.2014.05.018

[16] B.-N. Guo and F. Qi, On inequalities for the exponential and logarithmic functions and means, Malays. J. Math. Sci. 10 (2016), no. 1, 23-34.

[17] B.-N. Guo and F. Qi, Six proofs for an identity of the Lah numbers, Online J. Anal. Comb. 10 (2015), 5 pages.

[18] B.-N. Guo and F. Qi, Some identities and an explicit formula for Bernoulli and Stirling numbers, J. Comput. Appl. Math. 255 (2014), 568-579; available online at https://doi. org/10.1016/j.cam.2013.06.020

[19] B.-N. Guo and F. Qi, Some inequalities and absolute monotonicity for modified Bessel functions of the first kind, Commun. Korean Math. Soc. 31 (2016), no. 2, 355-363; available online at https://doi.org/10.4134/CKMS.2016.31.2.355

[20] F. T. Howard, A special class of Bell polynomials, Math. Comp. 35 (1980), no. 151, 977-989; available online at https://doi.org/10.2307/2006208

[21] F. T. Howard, Associated Stirling numbers, Fibonacci Quart. 18 (1980), no. 4, 303-315.

[22] K. K. Kataria and P. Vellaisamy, Correlation between Adomian and partial exponential Bell polynomials, C. R. Math. Acad. Sci. Paris 355 (2017), no. 9, 929-936; available online at https://doi.org/10.1016/j.crma.2017.08.002

[23] V. V. Kruchinin, Derivation of Bell polynomials of the second kind, arXiv preprint (2011), available online at http://arxiv.org/abs/1104.5065

[24] J. C. Mason and D. C. Handscomb, Chebyshev Polynomials, Chapman \& Hall/CRC, Boca Raton, FL, 2003.

[25] P. Natalini and P. E. Ricci, Bell polynomials and modified Bessel functions of half-integral order, Appl. Math. Comput. 268 (2015), 270-274; available online at https://doi.org/10. 1016/j.amc.2015.06.069

[26] P. Natalini and P. E. Ricci, Higher order Bell polynomials and the relevant integer sequences, Appl. Anal. Discrete Math. 11 (2017), no. 2, 327-339; available online at https://doi.org/ 10.2298/AADM1702327N

[27] P. Natalini and P. E. Ricci, Remarks on Bell and higher order Bell polynomials and numbers, Cogent Math. 3 (2016), Art. ID 1220670, 15 pages; available online at https://doi.org/ 10.1080/23311835.2016.1220670.

[28] F. W. J. Olver, D. W. Lozier, R. F. Boisvert, and C. W. Clark (eds.), NIST Handbook of Mathematical Functions, Cambridge University Press, New York, 2010; available online at http://dlmf.nist.gov/

[29] F. Qi, A new formula for the Bernoulli numbers of the second kind in terms of the Stirling numbers of the first kind, Publ. Inst. Math. (Beograd) (N.S.) 100(114) (2016), 243-249; available online at https://doi.org/10.2298/PIM150501028Q

[30] F. Qi, A simple form for coefficients in a family of nonlinear ordinary differential equations, Adv. Appl. Math. Sci. 17 (2018), no. 8, 555-561.

[31] F. Qi, A simple form for coefficients in a family of ordinary differential equations related to the generating function of the Legendre polynomials, Adv. Appl. Math. Sci. 17 (2018), no. 11, 693-700.

[32] F. Qi, An explicit formula for the Bell numbers in terms of the Lah and Stirling numbers, Mediterr. J. Math. 13 (2016), no. 5, 2795-2800; available online at https://doi.org/10. 1007/s00009-015-0655-7

[33] F. Qi, An explicit formula of a sequence of polynomials, IAENG Int. J. Appl. Math. 50 (2020), no. 2, in press; available online at https://www.researchgate.net/publication/ 339136060

[34] F. Qi, Derivatives of tangent function and tangent numbers, Appl. Math. Comput. 268 (2015), 844-858; available online at https://doi.org/10.1016/j.amc.2015.06.123

[35] F. Qi, Determinantal expressions and recurrence relations for Fubini and Eulerian polynomials, J. Interdiscip. Math. 22 (2019), no. 3, 317-335; available online at https: //doi.org/10.1080/09720502.2019.1624063

[36] F. Qi, Diagonal recurrence relations, inequalities, and monotonicity related to the Stirling numbers of the second kind, Math. Inequal. Appl. 19 (2016), no. 1, 313-323; available online at https://doi.org/10.7153/mia-19-23

[37] F. Qi, Diagonal recurrence relations for the Stirling numbers of the first kind, Contrib. Discrete Math. 11 (2016), no. 1, 22-30; available online at https://doi.org/10.11575/ cdm.v11i1.62389 
[38] F. Qi, Explicit formulas for computing Bernoulli numbers of the second kind and Stirling numbers of the first kind, Filomat 28 (2014), no. 2, 319-327; available online at https: //doi.org/10.2298/FIL14023190

[39] F. Qi, Explicit formulas for the convolved Fibonacci numbers, ResearchGate Preprint (2016), available online at https://doi.org/10.13140/RG.2.2.36768.17927

[40] F. Qi, Integral representations for multivariate logarithmic polynomials, J. Comput. Appl. Math. 336 (2018), 54-62; available online at https://doi.org/10.1016/j.cam.2017.11. 047.

[41] F. Qi, Notes on several families of differential equations related to the generating function for the Bernoulli numbers of the second kind, Turkish J. Anal. Number Theory 6 (2018), no. 2, 40-42; available online at https://doi.org/10.12691/tjant-6-2-1

[42] F. Qi, On multivariate logarithmic polynomials and their properties, Indag. Math. 29 (2018), no. 5, 1179-1192; available online at https://doi.org/10.1016/j.indag.2018.04.002

[43] F. Qi, Properties of modified Bessel functions and completely monotonic degrees of differences between exponential and trigamma functions, Math. Inequal. Appl. 18 (2015), no. 2, 493-518; available online at http://dx.doi.org/10.7153/mia-18-37.

[44] F. Qi, Simple forms for coefficients in two families of ordinary differential equations, Glob. J. Math. Anal. 6 (2018), no. 1, 7-9; available online at https://doi.org/10.14419/gjma. v6i1.9778

[45] F. Qi, Simplification of coefficients in two families of nonlinear ordinary differential equations, Turkish J. Anal. Number Theory 6 (2018), no. 4, 116-119; available online at https://doi.org/10.12691/tjant-6-4-2

[46] F. Qi, Simplifying coefficients in a family of nonlinear ordinary differential equations, Acta Comment. Univ. Tartu. Math. 22 (2018), no. 2, 293-297; available online at https://doi. org/10.12697/ACUTM.2018.22.24.

[47] F. Qi, Simplifying coefficients in a family of ordinary differential equations related to the generating function of the Laguerre polynomials, Appl. Appl. Math. 13 (2018), no. 2, $750-755$.

[48] F. Qi, Simplifying coefficients in a family of ordinary differential equations related to the generating function of the Mittag-Leffler polynomials, Korean J. Math. 27 (2019), no. 2, 417-423; available online at https://doi.org/10.11568/kjm.2019.27.2.417

[49] F. Qi, Simplifying coefficients in differential equations related to generating functions of reverse Bessel and partially degenerate Bell polynomials, Bol. Soc. Paran. Mat. 39 (2021), no. 4, in press; available online at http://dx.doi.org/10.5269/bspm.41758

[50] F. Qi, Some inequalities for the Bell numbers, Proc. Indian Acad. Sci. Math. Sci. 127 (2017), no. 4, 551-564; available online at https://doi.org/10.1007/s12044-017-0355-2

[51] F. Qi, Some inequalities and an application of exponential polynomials, Math. Inequal. Appl. 23 (2020), no. 1, 123-135; available online at https://doi.org/10.7153/mia-2020-23-10

[52] F. Qi and C. Berg, Complete monotonicity of a difference between the exponential and trigamma functions and properties related to a modified Bessel function, Mediterr. J. Math. 10 (2013), no. 4, 1685-1696; available online at http://dx.doi.org/10.1007/ s00009-013-0272-2.

[53] F. Qi and R. J. Chapman, Two closed forms for the Bernoulli polynomials, J. Number Theory 159 (2016), 89-100; available online at https://doi.org/10.1016/j.jnt.2015.07. 021

[54] F. Qi and V. Čerňanová, Some discussions on a kind of improper integrals, Int. J. Anal. Appl. 11 (2016), no. 2, 101-109.

[55] F. Qi, V. Čerňanová, and Y. S. Semenov, Some tridiagonal determinants related to central Delannoy numbers, the Chebyshev polynomials, and the Fibonacci polynomials, Politehn. Univ. Bucharest Sci. Bull. Ser. A Appl. Math. Phys. 81 (2019), no. 1, 123-136.

[56] F. Qi, V. Čerňanová, X.-T. Shi, and B.-N. Guo, Some properties of central Delannoy numbers, J. Comput. Appl. Math. 328 (2018), 101-115; available online at https: //doi.org/10.1016/j.cam.2017.07.013

[57] F. Qi and J. Gélinas, Revisiting Bouvier's paper on tangent numbers, Adv. Appl. Math. Sci. 16 (2017), no. 8, 275-281.

[58] F. Qi and B.-N. Guo, A closed form for the Stirling polynomials in terms of the Stirling numbers, Tbilisi Math. J. 10 (2017), no. 4, 153-158; available online at https://doi.org/ 10.1515/tmj-2017-0053

[59] F. Qi and B.-N. Guo, A diagonal recurrence relation for the Stirling numbers of the first kind, Appl. Anal. Discrete Math. 12 (2018), no. 1, 153-165; available online at https: //doi.org/10.2298/AADM170405004Q 
[60] F. Qi and B.-N. Guo, Alternative proofs of a formula for Bernoulli numbers in terms of Stirling numbers, Analysis (Berlin) 34 (2014), no. 3, 311-317; available online at https: //doi.org/10.1515/anly-2014-0003

[61] F. Qi and B.-N. Guo, An explicit formula for derivative polynomials of the tangent function, Acta Univ. Sapientiae Math. 9 (2017), no. 2, 348-359; available online at https://doi.org/ 10.1515/ausm-2017-0026

[62] F. Qi and B.-N. Guo, Explicit and recursive formulas, integral representations, and properties of the large Schröder numbers, Kragujevac J. Math. 41 (2017), no. 1, 121-141; available online at https://doi.org/10.5937/KgJMath1701121F

[63] F. Qi and B.-N. Guo, Explicit formulas and recurrence relations for higher order Eulerian polynomials, Indag. Math. 28 (2017), no. 4, 884-891; available online at https://doi.org/ 10.1016/j.indag.2017.06.010

[64] F. Qi and B.-N. Guo, Explicit formulas for derangement numbers and their generating function, J. Nonlinear Funct. Anal. 2016, Article ID 45, 10 pages.

[65] F. Qi and B.-N. Guo, Explicit formulas for special values of the Bell polynomials of the second kind and for the Euler numbers and polynomials, Mediterr. J. Math. 14 (2017), no. 3, Art. 140, 14 pages; available online at https://doi.org/10.1007/s00009-017-0939-1

[66] F. Qi and B.-N. Guo, On the sum of the Lah numbers and zeros of the Kummer confluent hypergeometric function, Acta Univ. Sapientiae Math. 10 (2018), no. 1, 125-133; available online at https://doi.org/10.2478/ausm-2018-0011

[67] F. Qi and B.-N. Guo, Several explicit and recursive formulas for generalized Motzkin numbers, AIMS Math. 5 (2020), no. 2, 1333-1345; available online at https://doi.org/10.3934/ math. 2020091

[68] F. Qi and B.-N. Guo, Some properties and generalizations of the Catalan, Fuss, and FussCatalan numbers, Chapter 5 in Mathematical Analysis and Applications: Selected Topics, First Edition, 101-133; Edited by Michael Ruzhansky, Hemen Dutta, and Ravi P. Agarwal; Published by John Wiley \& Sons, Inc. May 2018; available online at https://doi.org/10. 1002/9781119414421.ch5

[69] F. Qi and B.-N. Guo, Some properties of the average numbers of comparisons used by the quicksort, J. Math. Anal. 8 (2017), no. 2, 123-128.

[70] F. Qi and B.-N. Guo, Some properties of the Hermite polynomials, Georgian Math. J. 29 (2022), in press; available online at https://doi.org/10.20944/preprints201611.0145.v1

[71] F. Qi and B.-N. Guo, The reciprocal of the weighted geometric mean is a Stieltjes function, Bol. Soc. Mat. Mex. (3) 24 (2018), no. 1, 181-202; available online at https://doi.org/10. 1007/s40590-016-0151-5

[72] F. Qi and B.-N. Guo, Viewing some ordinary differential equations from the angle of derivative polynomials, Iran. J. Math. Sci. Inform. 15 (2020), no. 2, in press; available online at https://www.researchgate.net/publication/324966027.

[73] F. Qi, D. Lim, and B.-N. Guo, Explicit formulas and identities for the Bell polynomials and a sequence of polynomials applied to differential equations, Rev. R. Acad. Cienc. Exactas Fís. Nat. Ser. A Mat. RACSAM 113 (2019), no. 1, 1-9; available online at https://doi. org/10.1007/s13398-017-0427-2

[74] F. Qi, D. Lim, and B.-N. Guo, Some identities related to Eulerian polynomials and involving the Stirling numbers, Appl. Anal. Discrete Math. 12 (2018), no. 2, 467-480; available online at https://doi.org/10.2298/AADM171008014Q

[75] F. Qi, D. Lim, and Y.-H. Yao, Notes on two kinds of special values for the Bell polynomials of the second kind, Miskolc Math. Notes 20 (2019), no. 1, 465-474; available online at https://doi.org/10.18514/MMN.2019.2635

[76] F. Qi and A.-Q. Liu, Alternative proofs of some formulas for two tridiagonal determinants, Acta Univ. Sapientiae Math. 10 (2018), no. 2, 287-297; available online at https://doi. org/10.2478/ausm-2018-0022

[77] F. Qi, A.-Q. Liu, and D. Lim, Explicit expressions related to degenerate Cauchy numbers and their generating function, Mathematical Modelling, Applied Analysis and Computation, 41-52, Springer Proc. Math. Stat., 272, Springer, Singapore, 2019; available online at https : //doi.org/10.1007/978-981-13-9608-3_2

[78] F. Qi, P. Natalini, and P. E. Ricci, Recurrences of Stirling and Lah numbers via second kind Bell polynomials, Discrete Math. Lett. 3 (2020), 31-36.

[79] F. Qi, D.-W. Niu, and B.-N. Guo, Simplification of coefficients in differential equations associated with higher order Frobenius-Euler numbers, Tatra Mt. Math. Publ. 72 (2018), 67-76; available online at https://doi.org/10.2478/tmmp-2018-0022

[80] F. Qi, D.-W. Niu, and B.-N. Guo, Simplifying coefficients in differential equations associated with higher order Bernoulli numbers of the second kind, AIMS Math. 4 (2019), no. 2, 170-175; available online at https://doi.org/10.3934/Math.2019.2.170 
[81] F. Qi, D.-W. Niu, and B.-N. Guo, Some identities for a sequence of unnamed polynomials connected with the Bell polynomials, Rev. R. Acad. Cienc. Exactas Fís. Nat. Ser. A Math. RACSAM 113 (2019), no. 2, 557-567; available online at https://doi.org/10.1007/ s13398-018-0494-z

[82] F. Qi, D.-W. Niu, and D. Lim, Notes on explicit and inversion formulas for the Chebyshev polynomials of the first two kinds, Miskolc Math. Notes 20 (2019), no. 2, 1129-1137; available online at https://doi.org/10.18514/MMN. 2019.2976

[83] F. Qi, D.-W. Niu, D. Lim, and B.-N. Guo, Closed formulas and identities for the Bell polynomials and falling factorials, Contrib. Discrete Math. 15 (2020), no. 1, 163-174; available online at https://doi.org/10.11575/cdm.v15i1.68111

[84] F. Qi, D.-W. Niu, D. Lim, and B.-N. Guo, Some properties and an application of multivariate exponential polynomials, Math. Methods Appl. Sci. 43 (2020), no. 6, 2967-2983; available online at https://doi.org/10.1002/mma.6095

[85] F. Qi, D.-W. Niu, D. Lim, and Y.-H. Yao, Special values of the Bell polynomials of the second kind for some sequences and functions, HAL preprint (2018), available online at https://hal.archives-ouvertes.fr/hal-01766566

[86] F. Qi, X.-T. Shi, and B.-N. Guo, Two explicit formulas of the Schröder numbers, Integers 16 (2016), Paper No. A23, 15 pages.

[87] F. Qi, X.-T. Shi, and F.-F. Liu, Several identities involving the falling and rising factorials and the Cauchy, Lah, and Stirling numbers, Acta Univ. Sapientiae Math. 8 (2016), no. 2, 282-297; available online at https://doi.org/10.1515/ausm-2016-0019

[88] F. Qi, X.-T. Shi, F.-F. Liu, and D. V. Kruchinin, Several formulas for special values of the Bell polynomials of the second kind and applications, J. Appl. Anal. Comput. 7 (2017), no. 3, 857-871; available online at https://doi.org/10.11948/2017054

[89] F. Qi, X.-T. Shi, F.-F. Liu, and Z.-H. Yang, A double inequality for an integral mean in terms of the exponential and logarithmic means, Period. Math. Hungar. 75 (2017), no. 2, 180-189; available online at http://dx.doi.org/10.1007/s10998-016-0181-9

[90] F. Qi and S.-H. Wang, Complete monotonicity, completely monotonic degree, integral representations, and an inequality related to the exponential, trigamma, and modified Bessel functions, Glob. J. Math. Anal. 2 (2014), no. 3, 91-97; available online at http: //dx.doi.org/10.14419/gjma.v2i3.2919

[91] F. Qi, J.-L. Wang, and B.-N. Guo, Simplifying and finding ordinary differential equations in terms of the Stirling numbers, Korean J. Math. 26 (2018), no. 4, 675-681; available online at https://doi.org/10.11568/kjm.2018.26.4.675

[92] F. Qi, G.-S. Wu, and B.-N. Guo, An alternative proof of a closed formula for central factorial numbers of the second kind, Turkish J. Anal. Number Theory 7 (2019), no. 2, 56-58; available online at https://doi.org/10.12691/tjant-7-2-5

[93] F. Qi and Y.-H. Yao, Simplifying coefficients in differential equations for generating function of Catalan numbers, J. Taibah Univ. Sci. 13 (2019), no. 1, 947-950; available online at https://doi.org/10.1080/16583655.2019.1663782

[94] F. Qi, S.-W. Yao, and B.-N. Guo, Arithmetic means for a class of functions and the modified Bessel functions of the first kind, Mathematics 7 (2019), no. 1, Art. 60, 14 pages; available online at https://doi.org/10.3390/math7010060

[95] F. Qi and X.-J. Zhang, An integral representation, some inequalities, and complete monotonicity of the Bernoulli numbers of the second kind, Bull. Korean Math. Soc. 52 (2015), no. 3, 987-998; available online at https://doi.org/10.4134/BKMS.2015.52.3.987

[96] F. Qi and J.-L. Zhao, Some properties of the Bernoulli numbers of the second kind and their generating function, Bull. Korean Math. Soc. 55 (2018), no. 6, 1909-1920; available online at https://doi.org/10.4134/BKMS.b180039

[97] F. Qi and M.-M. Zheng, Explicit expressions for a family of the Bell polynomials and applications, Appl. Math. Comput. 258 (2015), 597-607; available online at https://doi. org/10.1016/j.amc.2015.02.027

[98] F. Qi, Q. Zou, and B.-N. Guo, The inverse of a triangular matrix and several identities of the Catalan numbers, Appl. Anal. Discrete Math. 13 (2019), no. 2, 518-541; available online at https://doi.org/10.2298/AADM190118018Q

[99] J. Quaintance and H. W. Gould, Combinatorial Identities for Stirling Numbers, the unpublished notes of H. W. Gould, with a foreword by George E. Andrews, World Scientific Publishing Co. Pte. Ltd., Singapore, 2016

[100] C.-F. Wei and B.-N. Guo, Complete monotonicity of functions connected with the exponential function and derivatives, Abstr. Appl. Anal. 2014 (2014), Article ID 851213, 5 pages; available online at https://doi.org/10.1155/2014/851213

[101] C.-F. Wei and F. Qi, Several closed expressions for the Euler numbers, J. Inequal. Appl. 2015, 2015:219, 8 pages; available online at https://doi.org/10.1186/s13660-015-0738-9 
[102] C. S. Withers and S. Nadarajah, Moments and cumulants for the complex Wishart, J. Multivariate Anal. 112 (2012), 242-247.

[103] C. S. Withers and S. Nadarajah, Multivariate Bell polynomials, Int. J. Comput. Math. 87 (2010), no. 11, 2607-2611; available online at https://doi.org/10.1080/ 00207160802702418

[104] C. S. Withers and S. Nadarajah, Multivariate Bell polynomials, series, chain rules, moments and inversion, Util. Math. 83 (2010), 133-140.

[105] C. S. Withers and S. Nadarajah, Multivariate Bell polynomials and their applications to powers and fractionary iterates of vector power series and to partial derivatives of composite vector functions, Appl. Math. Comput. 206 (2008), no. 2, 997-1004; available online at https://doi.org/10.1016/j.amc.2008.09.044

[106] A.-M. Xu and G.-D. Cen, Closed formulas for computing higher-order derivatives of functions involving exponential functions, Appl. Math. Comput. 270 (2015), 136-141; available online at https://doi.org/10.1016/j.amc.2015.08.051

[107] A.-M. Xu and Z.-D. Cen, Some identities involving exponential functions and Stirling numbers and applications, J. Comput. Appl. Math. 260 (2014), 201-207; available online at https://doi.org/10.1016/j.cam.2013.09.077

[108] J.-L. Zhao, Q.-M. Luo, B.-N. Guo, and F. Qi, Remarks on inequalities for the tangent function, Hacet. J. Math. Stat. 41 (2012), no. 4, 499-506.

[109] J.-L. Zhao, J.-L. Wang, and F. Qi, Derivative polynomials of a function related to the Apostol-Euler and Frobenius-Euler numbers, J. Nonlinear Sci. Appl. 10 (2017), no. 4, 1345-1349; available online at https://doi.org/10.22436/jnsa.010.04.06

[110] Z.-Z. Zhang and J.-Z. Yang, Notes on some identities related to the partial Bell polynomials, Tamsui Oxf. J. Inf. Math. Sci. 28 (2012), no. 1, 39-48.

[111] J.-L. Zhao and F. Qi, Two explicit formulas for the generalized Motzkin numbers, J. Inequal. Appl. 2017, 2017:44, 8 pages; available online at https://doi.org/10.1186/ s13660-017-1313-3

(Qi) Institute of Mathematics, Henan Polytechnic University, Jiaozuo 454010, Henan, China; College of Mathematics and Physics, Inner Mongolia University for Nationalities, Tongliao 028043, Inner Mongolia, China; School of Mathematical Sciences, Tianjin Polytechnic University, Tianjin 300387, China

Email address: qifeng618@gmail.com, qifeng618@hotmail.com, qifeng618@qq.com $U R L:$ https://qifeng618.wordpress.com, https://orcid.org/0000-0001-6239-2968

(Niu) Department of Science, Henan University of Animal Husbandry and Economy, Zhengzhou 450046, Henan, China

Email address: nnddww@gmail.com

URL: http://orcid.org/0000-0003-4033-7911

(Lim) Department of Mathematics Education, Andong National University, Andong 36729, Republic OF KoreA

Email address: dgrim84@gmail.com, dklim@andong.ac.kr

$U R L:$ https://orcid.org/0000-0002-0928-8480

(Yao) School of Mathematical Sciences, Tianjin Polytechnic University, Tianjin 300387, CHINA

Email address: yaoyonghong@aliyun.com

$U R L$ : https ://orcid.org/0000-0002-0452-785X 